\title{
Super Duality and Homology of Unitarizable Modules of Lie Algebras
}

\author{
by
}

Po-Yi Huang, Ngau LAm and Tze-Ming To

\begin{abstract}
The $\mathfrak{u}$-homology formulas for unitarizable modules at negative levels over classical Lie algebras of infinite rank of types $\mathfrak{g l}(n), \mathfrak{s p}(2 n)$ and $\mathfrak{s o}(2 n)$ are obtained. As a consequence, we recover Enright's formulas for three Hermitian symmetric pairs of classical types, $(S U(p, q), S U(p) \times S U(q)),(S p(2 n), U(n))$ and $\left(S O^{*}(2 n), U(n)\right)$.
\end{abstract}

2010 Mathematics Subject Classification: Primary 17B10, 17B56, $17 \mathrm{~B} 65$.

Keywords: super duality, homology, unitarizable module.

\section{$\S 1$. Introduction}

In analogy to Kostant's $\mathfrak{u}$-cohomology formulas [Ko], Enright establishes similar formulas [E] for unitarizable highest weight modules of Hermitian symmetric pairs in terms of certain complicated subsets of the Weyl groups. The argument there is intricate and involves several equivalences of categories and non-trivial combinatorics of the Weyl groups. Kostant's formula can be rephrased by saying that the Kazhdan-Lusztig polynomials associated to finite-dimensional modules are monomials. The same statement is true by Enright's formulas for unitarizable highest weight modules. Except for the resemblance of the formulas, there was no obvious connection between Enright's and Kostant's formulas.

Communicated by M. Kashiwara. Received May 10, 2010. Revised October 22, 2010 and January 12,2011

P.-Y. Huang: Department of Mathematics and National Center for Sciences (South), National Cheng Kung University, Tainan, Taiwan 701;

e-mail: pyhuang@mail.ncku.edu.tw

N. Lam: Department of Mathematics and National Center for Sciences (South),

National Cheng Kung University, Tainan, Taiwan 701;

e-mail: nlam@mail.ncku.edu.tw

T.-M. To: Department of Mathematics, National Changhua University of Education,

Changhua, Taiwan 500;

e-mail: matotm@cc.ncue.edu.tw

(C) 2012 Research Institute for Mathematical Sciences, Kyoto University. All rights reserved. 
However, the modules appearing in the Howe duality at negative levels [W, H1, H2] over classical Lie algebras of infinite rank are unitarizable modules (cf. [EHW], see also Proposition 2.6 and Remark 2.7 below) and the character formulas for these modules can be obtained by applying the involution of the ring of symmetric functions with infinite variables, which sends the elementary symmetric functions to the complete symmetric functions, to the characters for the corresponding integrable modules over the respective Lie algebras (cf. [CK, CKW]). Remarkably, the $\mathfrak{u}$-homology groups of these modules are also dictated by those of the corresponding integrable modules [CK, CKW]. The correspondence between $\mathfrak{u}$-homology groups of integrable modules at positive levels and $\mathfrak{u}$-homology groups of unitarizable modules (at negative levels) over the respective Lie algebras can be elucidated in terms of the so called super duality [CWZ, CW], established in $[\mathrm{BrS}, \mathrm{CL}, \mathrm{CLW}]$. Before [CLW] there was no explanation of the similarity of these two different $\mathfrak{u}$-homology groups. Super duality gives a first conceptual explanation of this similarity [CLW, Theorem 4.13].

To the best of our knowledge, there has been no proof of Enright's formulas different from the original one. In this paper, we give a proof of Enright's homology formulas for unitarizable modules by using Kostant's formulas and super duality. The $\mathfrak{u}$-homology formulas (see Theorem 4.4 below) for unitarizable modules over classical Lie algebras of infinite rank of types $\mathfrak{g l}(n), \mathfrak{s p}(2 n)$ and $\mathfrak{s o}(2 n)$ are obtained by combinatorial methods. The proof involves relating the combinatorial data of Kostant's formulas for integrable modules over corresponding Lie algebras, that are determined by the super duality, to the data of the Lie algebras under consideration. By applying the truncation functors (cf. [CLW, Section 3.4]) to the $\mathfrak{u}$-homology formulas (see also Section 2.4 below), we recover Enright's formulas for three Hermitian symmetric pairs of classical types, $(S U(p, q), S U(p) \times S U(q))$, $(S p(2 n), U(n))$ and $\left(S O^{*}(2 n), U(n)\right)$. However, for $\mathfrak{s o}(2 n)$, our method can only partially recover Enright's formula for some unitarizable highest weight cases.

The paper is organized as follows. In Section 2, we review and set up notation for the classical Lie algebras of finite and infinite rank. We describe the unitarizable highest weight modules considered in this paper. Combinatorial descriptions of Weyl groups are also given in that section. In Section 3, we compare the actions of certain subsets of Weyl groups on certain numerical data associated with the highest weights. In Section 4, homology formulas for unitarizable modules over Lie algebras of infinite rank are proved. In Section 5, Enright's homology formulas are proved.

We shall use the following notation throughout this article. The symbols $\mathbb{Z}$, $\mathbb{N}$, and $\mathbb{Z}_{+}$stand for the sets of all integers, of positive integers and of non-negative 
integers, respectively. We set $\mathbb{Z}^{*}:=\mathbb{Z} \backslash\{0\}$. For a partition $\lambda$, we denote by $\lambda^{\prime}$ the transpose partition of $\lambda$. Finally all vector spaces, algebras, tensor products etc. are over the field $\mathbb{C}$ of complex numbers.

\section{§2. Preliminaries}

\section{$\S 2.1$. Classical Lie algebras of infinite rank}

In this subsection we review and fix notation for Lie algebras of interest in this paper. For details we refer to [K, W, CK, CLW].

2.1.1. The Lie algebra $\mathfrak{a}_{\infty}$. Let $\mathbb{C}^{\infty}$ be the vector space over $\mathbb{C}$ with an ordered basis $\left\{e_{i} \mid i \in \mathbb{Z}\right\}$ so that an element in $\operatorname{End}\left(\mathbb{C}^{\infty}\right)$ may be identified with a matrix $\left(a_{i j}\right)(i, j \in \mathbb{Z})$. Let $E_{i j}$ be the matrix with 1 in the $i$-th row and $j$-th column and zeros elsewhere. Let $\stackrel{\mathfrak{a}}{\infty}_{\infty}$ denote the subalgebra of the Lie algebra $\operatorname{End}\left(\mathbb{C}^{\infty}\right)$ spanned by $E_{i j}$ with $i, j \in \mathbb{Z}$. Denote by $\mathfrak{a}_{\infty}:=\mathfrak{\mathfrak { a }}_{\infty} \oplus \mathbb{C} K$ the central extension of $\stackrel{\circ}{\infty}_{\infty}$ by the one-dimensional center $\mathbb{C} K$ given by the 2 -cocycle

$$
\tau(A, B):=\operatorname{Tr}([J, A] B),
$$

where $J=\sum_{i \leq 0} E_{i i}$ and $\operatorname{Tr}(C)$ is the trace of the matrix $C$. Observe that the cocycle $\tau$ is a coboundary. Indeed, there is an embedding $\iota_{\mathfrak{a}}$ from $\mathfrak{\mathfrak { a }}_{\infty}$ to $\mathfrak{a}_{\infty}$ defined by sending $A \in \stackrel{\circ}{\mathfrak{a}}_{\infty}$ to $A+\operatorname{Tr}(J A) K$ (cf. [CLW, Section 2.5]). It is clear that $\iota_{\mathfrak{a}}\left(\mathfrak{\mathfrak { a }}_{\infty}\right)$ is an ideal of $\mathfrak{a}_{\infty}$ and $\mathfrak{a}_{\infty}$ is a direct sum of the ideals $\iota_{\mathfrak{a}}\left(\dot{\mathfrak{a}}_{\infty}\right)$ and $\mathbb{C} K$. Note that $\iota_{\mathfrak{a}}\left(E_{i i}\right)=E_{i i}+K\left(\right.$ resp. $\left.E_{i i}\right)$ for $i \leq 0($ resp. $i \geq 1)$.

The Cartan subalgebra $\sum_{i \in \mathbb{Z}} \mathbb{C} E_{i i} \oplus \mathbb{C} K$ is denoted by $\mathfrak{h}_{\mathfrak{a}}$. By assigning degree 0 to the Cartan subalgebra and setting $\operatorname{deg} E_{i j}=j-i, \mathfrak{a}_{\infty}$ is equipped with a $\mathbb{Z}$-gradation $\mathfrak{a}_{\infty}=\bigoplus_{k \in \mathbb{Z}}\left(\mathfrak{a}_{\infty}\right)_{k}$. This leads to the following triangular decomposition:

$$
\mathfrak{a}_{\infty}=\left(\mathfrak{a}_{\infty}\right)_{+} \oplus\left(\mathfrak{a}_{\infty}\right)_{0} \oplus\left(\mathfrak{a}_{\infty}\right)_{-},
$$

where $\left(\mathfrak{a}_{\infty}\right)_{ \pm}=\bigoplus_{k \in \pm \mathbb{N}}\left(\mathfrak{a}_{\infty}\right)_{k}$ and $\left(\mathfrak{a}_{\infty}\right)_{0}=\mathfrak{h}_{\mathfrak{a}}$.

The sets of simple coroots, simple roots and positive roots of $\mathfrak{a}_{\infty}$ are respectively

$$
\begin{aligned}
\Pi_{\mathfrak{a}}^{\vee} & =\left\{\beta_{i}^{\vee}:=E_{i i}-E_{i+1, i+1}+\delta_{i 0} K \mid i \in \mathbb{Z}\right\}, \\
\Pi_{\mathfrak{a}} & =\left\{\beta_{i}:=\epsilon_{i}-\epsilon_{i+1} \mid i \in \mathbb{Z}\right\}, \\
\Delta_{\mathfrak{a}}^{+} & =\left\{\epsilon_{i}-\epsilon_{j} \mid i<j, i, j \in \mathbb{Z}\right\},
\end{aligned}
$$

where $\epsilon_{i} \in \mathfrak{h}_{\mathfrak{a}}^{*}$ is determined by $\left\langle\epsilon_{i}, E_{j j}\right\rangle=\delta_{i j}$ and $\left\langle\epsilon_{i}, K\right\rangle=0$. We also let $\vartheta_{\mathfrak{a}} \in \mathfrak{h}_{\mathfrak{a}}^{*}$ be defined by $\left\langle\vartheta_{\mathfrak{a}}, K\right\rangle=1$ and $\left\langle\vartheta_{\mathfrak{a}}, E_{j j}\right\rangle=0$ for all $j \in \mathbb{Z}$. Let $\rho_{\mathfrak{a}} \in \mathfrak{h}_{\mathfrak{a}}^{*}$ be determined by $\left\langle\rho_{\mathfrak{a}}, E_{j j}\right\rangle=-j$ for all $j \in \mathbb{Z}$, and $\left\langle\rho_{\mathfrak{a}}, K\right\rangle=0$, so that we have $\left\langle\rho_{\mathfrak{a}}, \alpha_{i}^{\vee}\right\rangle=1$ for all $i \in \mathbb{Z}$. 
2.1.2. The Lie algebras $\mathfrak{c}_{\infty}$ and $\mathfrak{d}_{\infty}$. For $\mathfrak{g}=\mathfrak{c}, \mathfrak{d}$, let $\mathfrak{g}_{\infty}$ be the subalgebra of $\stackrel{\circ}{\infty}_{\infty}$ preserving the following bilinear form on $\mathbb{C}^{\infty}$ :

$$
\left(e_{i} \mid e_{j}\right)=\left\{\begin{array}{ll}
(-1)^{i} \delta_{i, 1-j} & \text { if } \mathfrak{g}=\mathfrak{c}, \\
\delta_{i, 1-j} & \text { if } \mathfrak{g}=\mathfrak{d},
\end{array} \quad i, j \in \mathbb{Z}\right.
$$

Let $\mathfrak{g}_{\infty}=\grave{\mathfrak{g}}_{\infty} \oplus \mathbb{C} K$ be the central extension of $\stackrel{\mathfrak{g}}{\infty}_{\infty}$ determined by the restriction of the two-cocycle (2.1). Then $\mathfrak{g}_{\infty}$ has a natural $\mathbb{Z}$-gradation and a triangular decomposition induced from $\mathfrak{a}_{\infty}$ with $\left(\mathfrak{g}_{\infty}\right)_{n}=\mathfrak{g}_{\infty} \cap\left(\mathfrak{a}_{\infty}\right)_{n}$ for $n \in \mathbb{Z}$. Similar to the $\mathfrak{a}_{\infty}$ case, the cocycle is a coboundary. Indeed, there are embeddings $\iota_{\mathfrak{g}}$ from $\mathfrak{g}_{\infty}$ to $\mathfrak{g}_{\infty}$ defined by sending $A \in \mathfrak{\mathfrak { g }}_{\infty}$ to $A+\operatorname{Tr}(J A) K$ [CLW, Section 2.5]. It is clear that $\iota_{\mathfrak{g}}\left(\mathfrak{\mathfrak { g }}_{\infty}\right)$ is an ideal of $\mathfrak{g}_{\infty}$ and $\mathfrak{g}_{\infty}$ is a direct sum of the ideals $\iota_{\mathfrak{g}}\left(\dot{\mathfrak{g}}_{\infty}\right)$ and $\mathbb{C} K$. Note that $\iota_{\mathfrak{g}}\left(\widetilde{E}_{i}\right)=\widetilde{E}_{i}-K$ for $i \in \mathbb{N}$ where

$$
\widetilde{E}_{i}=E_{i i}-E_{1-i, 1-i}
$$

Note that $\left(\mathfrak{g}_{\infty}\right)_{0}=\sum_{i \in \mathbb{N}} \mathbb{C} \widetilde{E}_{i} \oplus \mathbb{C} K$ are Cartan subalgebras, which will be denoted by $\mathfrak{h}_{\mathfrak{g}}$. We let $\epsilon_{i} \in \mathfrak{h}_{\mathfrak{g}}^{*}$ be defined by $\left\langle\epsilon_{i}, \widetilde{E}_{j}\right\rangle=\delta_{i j}$ for $i, j \in \mathbb{N}$ and $\left\langle\epsilon_{i}, K\right\rangle=0$. Then the sets of positive roots of $\mathfrak{c}_{\infty}$ and $\mathfrak{d}_{\infty}$ are respectively

$$
\begin{aligned}
& \Delta_{\mathfrak{c}}^{+}=\left\{ \pm \epsilon_{i}-\epsilon_{j},-2 \epsilon_{i}(i, j \in \mathbb{N}, i<j)\right\} \\
& \Delta_{\mathfrak{d}}^{+}=\left\{ \pm \epsilon_{i}-\epsilon_{j}(i, j \in \mathbb{N}, i<j)\right\}
\end{aligned}
$$

Set

$$
\alpha_{0}^{\vee}=\left\{\begin{array}{ll}
-\widetilde{E}_{1}+K & \text { for } \mathfrak{c}_{\infty}, \\
-\widetilde{E}_{1}-\widetilde{E}_{2}+2 K & \text { for } \mathfrak{d}_{\infty},
\end{array} \quad \alpha_{0}= \begin{cases}-2 \epsilon_{1} & \text { for } \mathfrak{c}_{\infty}, \\
-\epsilon_{1}-\epsilon_{2} & \text { for } \mathfrak{d}_{\infty} .\end{cases}\right.
$$

The sets of simple coroots and simple roots of $\mathfrak{g}_{\infty}$ are respectively

$$
\begin{aligned}
\Pi_{\mathfrak{g}}^{\vee} & =\left\{\alpha_{0}^{\vee}, \alpha_{i}^{\vee}=\widetilde{E}_{i}-\widetilde{E}_{i+1}(i \in \mathbb{N})\right\}, \\
\Pi_{\mathfrak{g}} & =\left\{\alpha_{0}, \alpha_{i}=\epsilon_{i}-\epsilon_{i+1}(i \in \mathbb{N})\right\} .
\end{aligned}
$$

Let $\vartheta_{\mathfrak{g}} \in \mathfrak{h}_{\mathfrak{g}}^{*}$ be defined by $\left\langle\vartheta_{\mathfrak{g}}, \widetilde{E}_{i}\right\rangle=0$ for $i \in \mathbb{N}$ and $\left\langle\vartheta_{\mathfrak{g}}, K\right\rangle=r$ with $r=1$ (resp. $\frac{1}{2}$ ) for $\mathfrak{g}=\mathfrak{c}($ resp. $\mathfrak{d})$. We also let $\rho_{\mathfrak{g}} \in \mathfrak{h}_{\mathfrak{g}}^{*}$ be determined by

$$
\left\langle\rho_{\mathfrak{g}}, \widetilde{E}_{j}\right\rangle=\left\{\begin{array}{ll}
-j & \text { for } \mathfrak{g}=\mathfrak{c}, \\
-j+1 & \text { for } \mathfrak{g}=\mathfrak{d},
\end{array} \quad j \in \mathbb{N}, \quad \text { and } \quad\left\langle\rho_{\mathfrak{g}}, K\right\rangle=0 .\right.
$$

We have $\left\langle\rho_{\mathfrak{g}}, \alpha_{i}^{\vee}\right\rangle=1$ for $i \in \mathbb{N}$ and $\mathfrak{g}=\mathfrak{c}, \mathfrak{d}$.

2.1.3. Levi subalgebras. For $\mathfrak{g}=\mathfrak{a}, \mathfrak{c}, \mathfrak{d}$, let $\Delta_{\mathfrak{g}}:=\Delta_{\mathfrak{g}}^{+} \cup \Delta_{\mathfrak{g}}^{-}$, where $\Delta_{\mathfrak{g}}^{-}=-\Delta_{\mathfrak{g}}^{+}$. Then $\Delta_{\mathfrak{g}}$ is the set of roots of $\mathfrak{g}_{\infty}$. Let $\Delta_{\mathfrak{g}, c}^{ \pm}:=\Delta_{\mathfrak{g}}^{ \pm} \cap\left(\sum_{j \neq 0} \mathbb{Z} \alpha_{j}\right)$ and $\Delta_{\mathfrak{g}, n}^{ \pm}:=$ 
$\Delta_{\mathfrak{g}}^{ \pm} \backslash \Delta_{\mathfrak{g}, c}^{ \pm}$. Denote by $\mathfrak{g}_{\alpha}$ the root space corresponding to $\alpha \in \Delta_{\mathfrak{g}}$. Set

$$
\mathfrak{u}_{\mathfrak{g}}^{ \pm}:=\sum_{\alpha \in \Delta_{\mathfrak{g}, n}^{ \pm}} \mathfrak{g}_{\alpha}, \quad \mathfrak{l}_{\mathfrak{g}}:=\sum_{\alpha \in \Delta_{\mathfrak{g}, c}^{ \pm}} \mathfrak{g}_{\alpha} \oplus \mathfrak{h}_{\mathfrak{g}} .
$$

Then we have $\mathfrak{g}_{\infty}=\mathfrak{u}_{\mathfrak{g}}^{+} \oplus \mathfrak{l}_{\mathfrak{g}} \oplus \mathfrak{u}_{\mathfrak{g}}^{-}$. The Lie algebras $\mathfrak{l}_{\mathfrak{g}}$ and $\mathfrak{g}_{\infty}$ share the same Cartan subalgebra $\mathfrak{h}_{\mathfrak{g}}$. Moreover, $\mathfrak{l}_{\mathfrak{g}}$ has a triangular decomposition induced from $\mathfrak{g}_{\infty}$. For $\mu \in \mathfrak{h}_{\mathfrak{g}}^{*}$, we denote respectively by $L\left(\mathfrak{g}_{\infty}, \mu\right)$ and $L\left(\mathfrak{l}_{\mathfrak{g}}, \mu\right)$ the irreducible highest weight $\mathfrak{g}_{\infty}$-module and the $\mathfrak{l}_{\mathfrak{g}}$-module with highest weight $\mu$ with respect to the triangular decompositions.

For a root $\alpha \in \Delta_{\mathfrak{g}}, \mathfrak{g}=\mathfrak{a}, \mathfrak{c}, \mathfrak{d}$, define the reflection $\sigma_{\alpha}$ by

$$
\sigma_{\alpha}(\mu):=\mu-\left\langle\mu, \alpha^{\vee}\right\rangle \alpha, \quad \mu \in \mathfrak{h}_{\mathfrak{g}}^{*}
$$

Here and below, $\alpha^{\vee}$ denotes the coroot of the root $\alpha$. Let $I_{\mathfrak{a}}=\mathbb{Z}$ and $I_{\mathfrak{g}}=\mathbb{N}$ for $\mathfrak{g}=\mathfrak{c}, \mathfrak{d}$. For $j \in I_{\mathfrak{g}} \cup\{0\}$, let $\sigma_{j}=\sigma_{\alpha_{j}}$. Let $W_{\mathfrak{g}}$ be the subgroup of Aut $\left(\mathfrak{h}_{\mathfrak{g}}^{*}\right)$ generated by the reflections $\sigma_{j}$ with $j \in I_{\mathfrak{g}} \cup\{0\}$, i.e. $W_{\mathfrak{g}}$ is the Weyl group of $\mathfrak{g}_{\infty}$. For each $w \in W_{\mathfrak{g}}, \ell_{\mathfrak{g}}(w)$ denotes the length of $w$. We also define

$$
w \circ \mu:=w\left(\mu+\rho_{\mathfrak{g}}\right)-\rho_{\mathfrak{g}}, \quad \mu \in \mathfrak{h}_{\mathfrak{g}}^{*}, w \in W_{\mathfrak{g}} .
$$

Consider the subgroup $W_{\mathfrak{g}, 0}$ of $W_{\mathfrak{g}}$ generated by $\sigma_{j}$ with $j \neq 0$. Let $W_{\mathfrak{g}}^{0}$ denote the set of minimal length left coset representatives of $W_{\mathfrak{g}} / W_{\mathfrak{g}, 0}$ (cf. [V, Liu, Ku]). We have $W_{\mathfrak{g}}=W_{\mathfrak{g}}^{0} W_{\mathfrak{g}, 0}$. For $k \in \mathbb{Z}_{+}$, set

$$
W_{\mathfrak{g}, k}^{0}:=\left\{w \in W_{\mathfrak{g}}^{0} \mid \ell_{\mathfrak{g}}(w)=k\right\}
$$

Finally, for $\mathfrak{g}=\mathfrak{a}, \mathfrak{c}, \mathfrak{d}$, let $(\cdot \mid \cdot)$ be a bilinear form defined on a subspace of $\mathfrak{h}_{\mathfrak{g}}^{*}$ satisfying

$$
\left(\epsilon_{i} \mid \epsilon_{j}\right)=\delta_{i j}, \quad\left(\vartheta_{\mathfrak{g}} \mid \epsilon_{i}\right)=\left(\epsilon_{i} \mid \vartheta_{\mathfrak{g}}\right)=\left(\vartheta_{\mathfrak{g}} \mid \vartheta_{\mathfrak{g}}\right)=0 \quad \text { for } i, j \in I_{\mathfrak{g}}
$$

Recall that $I_{\mathfrak{a}}=\mathbb{Z}$ and $I_{\mathfrak{g}}=\mathbb{N}$ for $\mathfrak{g}=\mathfrak{c}, \mathfrak{d}$.

\section{$\S 2.2$. Finite-dimensional Lie algebras}

For the rest of the paper, let $\mathfrak{g}$ stand for $\mathfrak{a}, \mathfrak{c}, \mathfrak{d}$. We shall fix the following notation:

$$
\overline{\mathfrak{a}}:=\mathfrak{a}, \quad \overline{\mathfrak{c}}:=\mathfrak{d}, \quad \overline{\mathfrak{d}}:=\mathfrak{c} .
$$

Remark 2.1. For $\mathfrak{x}=\mathfrak{c}, \mathfrak{d}$, let $\mathfrak{g}^{\mathfrak{r}}$ and $\overline{\mathfrak{g}}^{\mathfrak{r}}$ be the Lie algebras defined in [CLW, Section 2] with $m=0$. Then $\mathfrak{c}_{\infty}=\mathfrak{g}^{\mathfrak{c}}, \mathfrak{d}_{\infty}=\mathfrak{g}^{\mathfrak{d}}, \overline{\mathfrak{c}}_{\infty} \cong \overline{\mathfrak{g}}^{\mathfrak{c}}$ and $\overline{\mathfrak{d}}_{\infty} \cong \overline{\mathfrak{g}}^{\mathfrak{d}}$. Note that $K$ maps to $-K$ under the isomorphisms $\overline{\mathfrak{c}}_{\infty} \cong \overline{\mathfrak{g}}^{\mathfrak{c}}$ and $\overline{\mathfrak{d}}_{\infty} \cong \overline{\mathfrak{g}}^{\mathfrak{d}}$. 
For $m, n \in \mathbb{N}$, the subalgebra of $\stackrel{\mathfrak{a}}{\infty}_{\infty}$ spanned by $E_{i j}$ with $1-m \leq i, j \leq n$, denoted by $\mathfrak{t}_{m, n} \mathfrak{a}$, is isomorphic to the general linear algebra $\mathfrak{g l}(m+n)$. The subalgebras $\left(\mathfrak{t}_{n, n} \mathfrak{a}\right) \cap \stackrel{\mathfrak{c}}{\infty}_{\infty}$ and $\left(\mathfrak{t}_{n, n} \mathfrak{a}\right) \cap \mathfrak{\mathfrak { d }}_{\infty}$ are isomorphic to the symplectic Lie algebra $\mathfrak{s p}(2 n)$ and the orthogonal Lie algebra $\mathfrak{s o}(2 n)$, denoted by $\mathfrak{t}_{n} \mathfrak{c}$ and $\mathfrak{t}_{n} \mathfrak{d}$ respectively. We shall drop the subscript of $\mathfrak{t}$ if there is no ambiguity.

For $\overline{\mathfrak{g}}=\mathfrak{a}, \mathfrak{c}, \mathfrak{d}$, the embeddings $\iota_{\overline{\mathfrak{g}}}^{\circ}$ restricted to $\mathfrak{t} \overline{\mathfrak{g}}$ are also denoted by $\iota_{\overline{\mathfrak{g}}}$. Let $\Delta_{\mathfrak{t} \overline{\mathfrak{g}}}^{+}$denote the set of positive roots of $\mathfrak{t} \overline{\mathfrak{g}}$ with respect to the triangular decomposition induced from $\overline{\mathfrak{g}}_{\infty}$. We also let $\Delta_{\mathfrak{t} \overline{\mathfrak{g}}}=\Delta_{\mathfrak{t} \overline{\mathfrak{g}}}^{+} \cup-\Delta_{\mathfrak{t} \overline{\mathfrak{g}}}^{+}$and $\Delta_{\mathfrak{t} \overline{\mathfrak{g}}, n}^{+}=\Delta_{\overline{\mathfrak{g}}, n}^{+} \cap \Delta_{\mathfrak{t} \overline{\mathfrak{g}}}^{+}$. Set $\mathfrak{h}_{\mathfrak{t} \overline{\mathfrak{g}}}=\mathfrak{h}_{\overline{\mathfrak{g}}} \cap \mathfrak{t} \overline{\mathfrak{g}}, \mathfrak{u}_{\mathfrak{t} \overline{\mathfrak{g}}}^{ \pm}=\mathfrak{u}_{\overline{\mathfrak{g}}}^{ \pm} \cap \mathfrak{t} \overline{\mathfrak{g}}$ and $\mathfrak{l}_{\mathfrak{t} \overline{\mathfrak{g}}}=\mathfrak{l}_{\overline{\mathfrak{g}}} \cap \mathfrak{t} \overline{\mathfrak{g}}$. Note that $\mathfrak{t} \overline{\mathfrak{g}}$ and $\mathfrak{l}_{\mathfrak{t} \overline{\mathfrak{g}}}$ share the same Cartan subalgebra $\mathfrak{h}_{\mathfrak{t} \mathfrak{g}}$. Moreover, $\mathfrak{l}_{\mathfrak{t} \overline{\mathfrak{g}}}$ has a triangular decomposition induced from $\mathfrak{t} \overline{\mathfrak{g}}$. For $\mu \in \mathfrak{h}_{\mathfrak{t} \mathfrak{g}}^{*}$, we denote respectively by $L(\mathfrak{t} \overline{\mathfrak{g}}, \mu)$ and $L\left(\mathfrak{l}_{\mathfrak{t} \overline{\mathfrak{g}}}, \mu\right)$ the irreducible highest weight $\mathfrak{t} \overline{\mathfrak{g}}$-module and the $\mathfrak{l}_{\mathfrak{t} \overline{\mathfrak{g}}}$-module with highest weight $\mu$ with respect to the triangular decompositions. For $\mu \in \mathfrak{h}_{\mathfrak{t} \overline{\mathfrak{g}}}^{*}, L\left(\mathfrak{l}_{\mathfrak{t} \overline{\mathfrak{g}}}, \mu\right)$ is extended to an $\left(\mathfrak{l}_{\mathfrak{t} \overline{\mathfrak{g}}}+\mathfrak{u}_{\mathfrak{t} \overline{\mathfrak{g}}}^{+}\right.$--module by letting $\mathfrak{u}_{\mathfrak{t} \overline{\mathfrak{g}}}^{+}$act trivially. Let $\mathfrak{p}_{\mathfrak{t} \overline{\mathfrak{g}}}=\mathfrak{l}_{\mathfrak{t} \overline{\mathfrak{g}}}+\mathfrak{u}_{\mathfrak{t} \overline{\mathfrak{g}}}^{+}$. Define as usual the parabolic Verma module with highest weight $\mu$ by

$$
N(\mathfrak{t} \overline{\mathfrak{g}}, \mu)=\operatorname{Ind}_{\mathfrak{p}_{\mathfrak{t} \overline{\mathfrak{g}}}}^{\mathfrak{t} \overline{\mathfrak{g}}} L\left(\mathfrak{l}_{\mathfrak{t} \overline{\mathfrak{g}}}, \mu\right) .
$$

The space $\mathfrak{h}_{\mathfrak{t} \mathfrak{g}}^{*}$ is spanned by $\epsilon_{i}$ with $1 \leq i \leq n$ (resp. $\left.1-m \leq i \leq n-1\right)$ for $\overline{\mathfrak{g}}=\mathfrak{c}, \mathfrak{d}$ (resp. $\mathfrak{a}$ ) and therefore $\mathfrak{h}_{\mathfrak{t} \mathfrak{g}}^{*}$ can be regarded as a subspace of $\mathfrak{h}_{\mathfrak{g}}^{*}$. Note that $\mathfrak{h}_{\mathfrak{t} \mathfrak{g}}^{*}$ is an invariant subspace of $\sigma_{i}$ for $1 \leq i \leq n$ (resp. $1-m \leq i \leq n-1$ ) for $\overline{\mathfrak{g}}=\mathfrak{c}$ or $\mathfrak{d}$ (resp. $\mathfrak{a})$. The restrictions of these $\sigma_{i}$ to $\mathfrak{h}_{\mathfrak{t} \mathfrak{g}}^{*}$ are also denoted by $\sigma_{i}$. Let $W_{\mathfrak{t} \bar{g}}$ be the subgroup of Aut $\left(\mathfrak{h}_{\mathfrak{t} \mathfrak{g}}^{*}\right)$ generated by these $\sigma_{i}$ s. Then $W_{\mathfrak{t} \overline{\mathfrak{g}}}$ is the Weyl group of $\mathfrak{t} \overline{\mathfrak{g}}$. For each $w \in W_{\mathfrak{t} \overline{\mathfrak{g}}}$ we let $\ell_{\mathfrak{t} \overline{\mathfrak{g}}}(w)$ denote the length of $w$. Consider the subgroup $W_{\mathfrak{t} \overline{\mathfrak{g}}, 0}$ of $W_{\mathfrak{t} \bar{g}}$ generated by $\sigma_{j}$ with $j \neq 0$. Let $W_{\mathfrak{t} \overline{\mathfrak{g}}}^{0}$ denote the set of minimal length representatives of the left coset space $W_{\mathfrak{t} \bar{g}} / W_{\mathbf{t} \bar{g}, 0}$ (cf. [Liu, Ku]). For $k \in \mathbb{Z}_{+}$, set $W_{\mathfrak{t} \mathfrak{g}, k}^{0}:=\left\{w \in W_{\mathfrak{t} \bar{g}}^{0} \mid \ell_{\mathfrak{t} \overline{\mathfrak{g}}}(w)=k\right\}$. We also define

$$
w \circ \mu:=w\left(\mu+\rho_{\mathfrak{t} \overline{\mathfrak{g}}}\right)-\rho_{\mathfrak{t} \overline{\mathfrak{g}}}, \quad \mu \in \mathfrak{h}_{\mathfrak{t} \overline{\mathfrak{g}}}^{*}, w \in W_{\mathfrak{t} \overline{\mathfrak{g}}} .
$$

Finally, let $\rho_{\mathfrak{t} \overline{\mathfrak{g}}}$ denote the half sum of the positive roots. Then $\rho_{\mathfrak{t} \overline{\mathfrak{g}}}(h)=\rho_{\overline{\mathfrak{g}}}(h)$ $\left(\right.$ resp. $\left.=\rho_{\mathfrak{a}}(h)+\frac{1}{2}(n-m+1)\right)$ for $h \in \mathfrak{h}_{\mathfrak{t} \overline{\mathfrak{g}}}$ with $\overline{\mathfrak{g}}=\mathfrak{c}, \mathfrak{d}(\operatorname{resp} . \overline{\mathfrak{g}}=\mathfrak{a})$.

\section{$\S 2.3$. Combinatorial descriptions of Weyl groups}

In this section, we present combinatorial descriptions of certain aspects of infinite Weyl groups $W_{\mathfrak{g}}$ (cf. [BB]). Recall that $\mathbb{Z}^{*}:=\mathbb{Z} \backslash\{0\}$.

Define $\phi_{\mathfrak{g}} \in \mathfrak{h}_{\mathfrak{g}}^{*}$ by

$$
\phi_{\mathfrak{g}}= \begin{cases}-\sum_{i \leq 0} \epsilon_{i} & \text { if } \mathfrak{g}=\mathfrak{a} \\ \sum_{i \in \mathbb{N}} \epsilon_{i} & \text { if } \mathfrak{g}=\mathfrak{c}, \mathfrak{d}\end{cases}
$$


Every $\lambda \in \mathfrak{h}_{\mathfrak{g}}^{*}$ can be uniquely represented by $\sum_{i \in I_{\mathfrak{g}}} \xi_{i} \epsilon_{i}+q \vartheta_{\mathfrak{g}}$ with $\xi_{i}, q \in \mathbb{C}$. For $\mathfrak{g}=\mathfrak{c}, \mathfrak{d}$ and $i \in \mathbb{N}$, we define $\epsilon_{-i}=-\epsilon_{i}$. It is easy to see by computing the action of $\sigma_{i}$ that the action of $W_{\mathfrak{g}}$ on $\mathfrak{h}_{\mathfrak{g}}^{*}$ is given by

$$
\sigma\left(\sum_{i \in \mathbb{Z}} \xi_{i} \epsilon_{i}+q \vartheta_{\mathfrak{a}}\right)=\sum_{i \leq 0}\left(\xi_{i}+q\right) \epsilon_{\tilde{\sigma}(i)}+\sum_{i>0} \xi_{i} \epsilon_{\tilde{\sigma}(i)}+q \phi_{\mathfrak{a}}+q \vartheta_{\mathfrak{a}} \quad \text { if } \mathfrak{g}=\mathfrak{a} ;
$$

$$
\sigma\left(\sum_{i \in \mathbb{N}} \xi_{i} \epsilon_{i}+q \vartheta_{\mathfrak{g}}\right)=\sum_{i \in \mathbb{N}}\left(\xi_{i}-q\left\langle\vartheta_{\mathfrak{g}}, K\right\rangle\right) \epsilon_{\tilde{\sigma}(i)}+q\left\langle\vartheta_{\mathfrak{g}}, K\right\rangle \phi_{\mathfrak{g}}+q \vartheta_{\mathfrak{g}} \quad \text { if } \mathfrak{g}=\mathfrak{c}, \mathfrak{d},
$$

where $\tilde{\sigma}$ is a permutation of $\mathbb{Z}$ (i.e. $\tilde{\sigma}$ is a bijection on $\mathbb{Z}$ satisfying $\tilde{\sigma}(j)=j$ for $|j| \gg 0$ ) for $\mathfrak{g}=\mathfrak{a}$ and $\tilde{\sigma}$ is a signed permutation of $\mathbb{Z}^{*}$ (i.e. $\tilde{\sigma}$ is a bijection on $\mathbb{Z}^{*}$ satisfying $\tilde{\sigma}(j)=j$ for $|j| \gg 0$ and $\tilde{\sigma}(-i)=-\tilde{\sigma}(i)$ for $\left.i \in \mathbb{Z}^{*}\right)$ for $\mathfrak{g}=\mathfrak{c}, \mathfrak{d}$. Therefore $\sigma \mapsto \tilde{\sigma}$ is a representation on $\mathbb{Z}$ and $\mathbb{Z}^{*}$ for $\mathfrak{g}=\mathfrak{a}$ and $\mathfrak{g}=\mathfrak{c}, \mathfrak{d}$, respectively. Moreover, these are faithful representations. It is clear that the image of $W_{\mathfrak{a}}$ in $\operatorname{Aut}(\mathbb{Z})$ is the set of permutations of $\mathbb{Z}$ and the image of $W_{\mathfrak{c}}\left(\right.$ resp. $\left.W_{\mathfrak{d}}\right)$ in $\operatorname{Aut}\left(\mathbb{Z}^{*}\right)$ is the set of signed (resp. even signed) permutations of $\mathbb{Z}^{*}$. A signed permutation $\tilde{\sigma}$ of $\mathbb{Z}^{*}$ is called even if $|\{i \in \mathbb{N} \mid \tilde{\sigma}(i)<0\}|$ is an even number. We shall identify $W_{\mathfrak{g}}$ with the image of $W_{\mathfrak{g}}$ in $\operatorname{Aut}(\mathbb{Z})\left(\operatorname{resp}\right.$. Aut $\left.\left(\mathbb{Z}^{*}\right)\right)$ in the case $\mathfrak{g}=\mathfrak{a}($ resp. $\mathfrak{c}, \mathfrak{d})$ for the rest of the paper. Note that for $i \in \mathbb{Z}, \tilde{\sigma}_{i}(i)=i+1, \tilde{\sigma}_{i}(i+1)=i$ and $\tilde{\sigma}_{i}(j)=j$ for all $j \neq i, i+1$. Also for $\mathfrak{g}=\mathfrak{c}, \mathfrak{d}$ and $i \in \mathbb{N}, \tilde{\sigma}_{i}(i)=i+1, \tilde{\sigma}_{i}(i+1)=i$ and $\tilde{\sigma}_{i}(j)=j$ for all $j \neq i, i+1$ while $\tilde{\sigma}_{0}(1)=-1$ (resp. -2$), \tilde{\sigma}_{0}(2)=2($ resp. -1$)$, and $\tilde{\sigma}_{0}(j)=j$ for all $j \geq 3$ for $\mathfrak{g}=\mathfrak{c}$ (resp. $\left.\mathfrak{d}\right)$. We shall use these representations in the rest of the paper and we shall simply write $\sigma(j)$ instead of $\tilde{\sigma}(j)$.

Recall that $\ell_{\mathfrak{g}}$ denotes the length function on $W_{\mathfrak{g}}$, and $W_{\mathfrak{g}}^{0}$ the set of minimal length left coset representatives of $W_{\mathfrak{g}} / W_{\mathfrak{g}, 0}$. We have

$$
W_{\mathfrak{g}}^{0}= \begin{cases}\left\{\sigma \in W_{\mathfrak{a}} \mid \sigma(i)<\sigma(j) \text { for } i<j \leq 0 \text { and } 0<i<j\right\} & \text { if } \mathfrak{g}=\mathfrak{a}, \\ \left\{\sigma \in W_{\mathfrak{g}} \mid \sigma(i)<\sigma(j) \text { for } 1 \leq i<j\right\} & \text { if } \mathfrak{g}=\mathfrak{c}, \mathfrak{d}\end{cases}
$$

(see e.g. [BB, Lemma 2.4.7 and Propositions 8.1.4 and 8.2.4]) and for $\sigma \in W_{\mathfrak{g}}^{0}$,

$$
\ell_{\mathfrak{g}}(\sigma)= \begin{cases}|\{(i, j) \in \mathbb{Z} \times \mathbb{Z} \mid i<j, \sigma(i)>\sigma(j)\}| & \text { if } \mathfrak{g}=\mathfrak{a} \\ |\{(i, j) \in \mathbb{N} \times \mathbb{N} \mid i \leq j, \sigma(-i)>\sigma(j)\}| & \text { if } \mathfrak{g}=\mathfrak{c} \\ |\{(i, j) \in \mathbb{N} \times \mathbb{N} \mid i<j, \sigma(-i)>\sigma(j)\}| & \text { if } \mathfrak{g}=\mathfrak{d}\end{cases}
$$

(see e.g. [BB, Corollaries 1.5.2, 8.1.1 and 8.2.1]). 
Lemma 2.2. For $\sigma \in W_{\mathfrak{c}}^{0}$ with $\sigma(i)<0$ for $i \leq j$, and $\sigma(i)>0$ for $i>j$, define $\bar{\sigma} \in W_{\mathfrak{d}}^{0}$ by

$$
\bar{\sigma}(i)= \begin{cases}\sigma(i)-1 & \text { if } i \leq j \\ 1 & \text { if } i=j+1 \text { and } j \text { is even, } \\ -1 & \text { if } i=j+1 \text { and } j \text { is odd, } \\ \sigma(i-1)+1 & \text { if } i \geq j+2 .\end{cases}
$$

For each $k \geq 0$, the map from $W_{\mathfrak{c}, k}^{0}$ to $W_{\mathfrak{d}, k}^{0}$ sending $\sigma$ to $\bar{\sigma}$ is a bijection.

Proof. By $(2.5)$, it is a bijection from $W_{\mathfrak{c}}^{0}$ to $W_{\mathfrak{d}}^{0}$. By $(2.6)$, we have $\ell_{\mathfrak{c}}(\sigma)=\ell_{\mathfrak{d}}(\bar{\sigma})$ for $\sigma \in W_{\mathfrak{c}}^{0}$. The lemma follows.

Let $\left\{\xi_{i}\right\}_{i \in \mathbb{N}}$ be a sequence of real numbers. Define $\xi_{-i}:=-\xi_{i}$ for $i \in \mathbb{N}$. For any strictly decreasing sequence $\left\{\xi_{i}\right\}_{i \in \mathbb{N}}$ of negative real numbers and $\sigma \in W_{\mathfrak{g}}^{0}$ with $\mathfrak{g}=\mathfrak{c}, \mathfrak{d}$, it is easy to see that $\left\{\xi_{\sigma(i)}\right\}_{i \in \mathbb{N}}$ is strictly decreasing. The following lemma follows from the definition of $\bar{\sigma}$.

Lemma 2.3. Let $\left\{\xi_{i}\right\}_{i \in \mathbb{N}}$ be a strictly decreasing sequence of negative real numbers. Define $\bar{\xi}_{i+1}=\xi_{i}$ for $i \in \mathbb{N}$ and $\bar{\xi}_{1}=0$. Then for all $\sigma \in W_{\mathfrak{c}}^{0}$, we have

$$
\left\{\xi_{\sigma(i)} \mid i \in \mathbb{N}\right\} \cup\{0\}=\left\{\bar{\xi}_{\bar{\sigma}(i)} \mid i \in \mathbb{N}\right\},
$$

where $\bar{\sigma}$ is defined in Lemma 2.2 .

\section{§2.4. Unitarizable highest weight modules}

Recall that $\mathfrak{g}$ stands for $\mathfrak{a}, \mathfrak{c}, \mathfrak{d}$, and $\overline{\mathfrak{a}}=\mathfrak{a}, \overline{\mathfrak{c}}=\mathfrak{d}$ and $\overline{\mathfrak{d}}=\mathfrak{c}$. In this subsection we classify the highest weights of irreducible unitarizable quasi-finite highest weight $\overline{\mathfrak{g}}_{\infty}$-modules with respect to the anti-linear anti-involution $\omega$ defined below.

For a partition $\lambda=\left(\lambda_{1}, \lambda_{2}, \ldots\right)$, the transpose partition of $\lambda$ is denoted by $\lambda^{\prime}=\left(\lambda_{1}^{\prime}, \lambda_{2}^{\prime}, \ldots\right)$. For $\mathfrak{g}=\mathfrak{c}, \mathfrak{d}$, a partition $\lambda$ and $d \in \mathbb{C}$, define

$$
\Lambda^{\mathfrak{g}}(\lambda, d):=\sum_{i \in \mathbb{N}} \lambda_{i}^{\prime} \epsilon_{i}+d \vartheta_{\mathfrak{g}} \in \mathfrak{h}_{\mathfrak{g}}^{*}, \quad \bar{\Lambda}^{\mathfrak{g}}(\lambda, d)=\sum_{i \in \mathbb{N}} \lambda_{i} \epsilon_{i}-\frac{d\left\langle\vartheta_{\mathfrak{g}}, K\right\rangle}{\left\langle\vartheta_{\overline{\mathfrak{g}}}, K\right\rangle} \vartheta_{\overline{\mathfrak{g}}} \in \mathfrak{h}_{\overline{\mathfrak{g}}}^{*}
$$

Let $\mathcal{D}(\mathfrak{g})$ denote the set of pairs $(\lambda, d)$ with $d \in \mathbb{Z}_{+}$satisfying $\lambda_{1}^{\prime} \leq d$ if $\mathfrak{g}=\mathfrak{c}$, and $\lambda_{1}^{\prime}+\lambda_{2}^{\prime} \leq d$ if $\mathfrak{g}=\mathfrak{d}$. For a pair $\lambda=\left(\lambda^{-}, \lambda^{+}\right)$of partitions and $d \in \mathbb{C}$, define $\Lambda^{\mathfrak{a}}(\lambda, d), \bar{\Lambda}^{\mathfrak{a}}(\lambda, d) \in \mathfrak{h}_{\mathfrak{a}}^{*}$ by

$$
\begin{aligned}
& \Lambda^{\mathfrak{a}}(\lambda, d)=-\sum_{i \in \mathbb{Z}_{+}}\left(\lambda^{-}\right)_{i+1}^{\prime} \epsilon_{-i}+\sum_{i \in \mathbb{N}}\left(\lambda^{+}\right)_{i}^{\prime} \epsilon_{i}+d \vartheta_{\mathfrak{a}} \\
& \bar{\Lambda}^{\mathfrak{a}}(\lambda, d)=-\sum_{i \in \mathbb{Z}_{+}} \lambda_{i+1}^{-} \epsilon_{-i}+\sum_{i \in \mathbb{N}} \lambda_{i}^{+} \epsilon_{i}-d \vartheta_{\mathfrak{a}}
\end{aligned}
$$

Let $\mathcal{D}(\mathfrak{a})$ denote the set of pairs $(\lambda, d)$ satisfying $d \in \mathbb{Z}_{+}$and $\left(\lambda^{-}\right)_{1}^{\prime}+\left(\lambda^{+}\right)_{1}^{\prime} \leq d$. 
Let $\mathfrak{k}$ be a Lie algebra equipped with an anti-linear anti-involution $\omega$, and let $V$ be a $\mathfrak{k}$-module. A Hermitian form $\langle\cdot \mid \cdot\rangle$ on $V$ is said to be contravariant if $\left\langle a v \mid v^{\prime}\right\rangle=\left\langle v \mid \omega(a) v^{\prime}\right\rangle$ for all $a \in \mathfrak{k}, v, v^{\prime} \in V$. A k -module equipped with a positive definite contravariant Hermitian form is called unitarizable. Assume that $\mathfrak{k}=\bigoplus_{j \in \mathbb{Z}} \mathfrak{k}_{j}$ (possibly $\operatorname{dim} \mathfrak{k}_{j}=\infty$ ) is a $\mathbb{Z}$-graded Lie algebra and $\mathfrak{k}_{0}$ is abelian. A graded $\mathfrak{k}$-module $M=\bigoplus_{j \in \mathbb{Z}} M_{j}$ is called quasi-finite if $\operatorname{dim} M_{j}<\infty$ for all $j \in \mathbb{Z}[\mathrm{KR}]$.

Remark 2.4. Let $V$ be a highest weight $\mathfrak{g}_{\infty}$-module with highest weight $\xi$. Using the arguments as in [LZ, Section 4], we find that $V$ is quasi-finite if and only if $\xi$ satisfies $\xi\left(E_{i i}\right)=0$ (resp. $\xi\left(\widetilde{E}_{i i}\right)=0$ ) for $|i| \gg 0$ (resp. $i \gg 0$ ) for $\mathfrak{g}=\mathfrak{a}$ (resp. $\mathfrak{c}, \mathfrak{d})$. Therefore every quasi-finite integrable highest weight $\mathfrak{g}_{\infty}$-module is of the form $L\left(\mathfrak{g}_{\infty}, \Lambda^{\mathfrak{g}}(\lambda, d)\right)$ for some $(\lambda, d) \in \mathcal{D}(\mathfrak{g})$.

Now we consider the anti-linear anti-involution $\omega$ on $\mathfrak{a}_{\infty}$ defined by

$$
\omega\left(E_{i j}\right)=\left\{\begin{array}{ll}
E_{j i} & \text { if } i, j \leq 0 \text { or } i, j>0, \\
-E_{j i} & \text { if } i>0, j \leq 0 \text { or } i \leq 0, j>0,
\end{array} \quad \text { and } \quad \omega(K)=K .\right.
$$

For $\mathfrak{g}=\mathfrak{c}, \mathfrak{d}$, the restriction of the anti-linear anti-involution $\omega$ on $\mathfrak{a}_{\infty}$ to $\mathfrak{g}_{\infty}$ gives an anti-linear anti-involution on $\mathfrak{g}_{\infty}$, which will also be denoted by $\omega$.

For $d \in \mathbb{C}$ and a pair $\lambda=\left(\lambda^{-}, \lambda^{+}\right)$of partitions with $\lambda_{n+1}^{+}=\lambda_{m+1}^{-}=0$, let $\Gamma_{\mathfrak{t a}}(\lambda, d)$ be the element in $\mathfrak{h}_{\mathfrak{t a}}^{*}$ determined by

$$
\Gamma_{\mathfrak{t} \overline{\mathfrak{a}}}(\lambda, d)=\sum_{i=1}^{m}\left(-d-\lambda_{i}^{-}\right) \epsilon_{-i+1}+\sum_{i=1}^{n} \lambda_{i}^{+} \epsilon_{i} .
$$

For $d \in \mathbb{C}$ and a partition $\lambda$ satisfying $\lambda_{n+1}=0$, let $\Gamma_{\mathfrak{t} \overline{\mathfrak{g}}}(\lambda, d)$ be the element in $h_{\mathfrak{t} \overline{\mathfrak{g}}}^{*}$ determined by

$$
\Gamma_{\mathfrak{t} \overline{\mathfrak{g}}}(\lambda, d)= \begin{cases}\sum_{i=1}^{n}\left(\lambda_{i}+d / 2\right) \epsilon_{i} & \text { for } \overline{\mathfrak{g}}=\mathfrak{c}, \\ \sum_{i=1}^{n}\left(\lambda_{i}+d\right) \epsilon_{i} & \text { for } \overline{\mathfrak{g}}=\mathfrak{d} .\end{cases}
$$

Let $\mathcal{D}_{\mathfrak{t}}(\mathfrak{g})$ denote the subset of $\mathcal{D}(\mathfrak{g})$ consisting of all elements $(\lambda, d)$ satisfying $\lambda_{n+1}=0$ for $\overline{\mathfrak{g}}=\mathfrak{c}, \mathfrak{d}$ (resp. $\lambda_{n+1}^{+}=0$ and $\lambda_{m+1}^{-}=0$ for $\overline{\mathfrak{g}}=\mathfrak{a}$ ).

Now we introduce the truncation functors [CLW, Section 3.4]. Let $M=$ $\bigoplus_{\beta} M_{\beta}$ be a semisimple $\mathfrak{h}_{\mathfrak{g}}$-module such that $M_{\beta}$ is the weight space of $M$ with weight $\beta$. The truncation functor $\mathfrak{t r}_{\mathfrak{t}}$ is defined by sending $M$ to $\bigoplus_{\nu} M_{\nu}$, summed over $\sum_{i=1-m}^{n} \mathbb{C} \epsilon_{i}+\mathbb{C} \vartheta_{\overline{\mathfrak{g}}}\left(\right.$ resp. $\left.\sum_{i=1}^{n} \mathbb{C} \epsilon_{i}+\mathbb{C} \vartheta_{\overline{\mathfrak{g}}}\right)$ for $\overline{\mathfrak{g}}=\mathfrak{a}($ resp. $\mathfrak{c}, \mathfrak{d})$. For $(\lambda, d) \in$ 
$\mathcal{D}(\mathfrak{g}), L\left(\overline{\mathfrak{g}}_{\infty}, \bar{\Lambda}^{\mathfrak{g}}(\lambda, d)\right)$ is a $\mathfrak{t} \overline{\mathfrak{g}}$-module through the embedding $\iota_{\mathfrak{\mathfrak { g }}}^{\circ}$ defined in Section 2.2. Then $\mathfrak{t r}_{\mathfrak{t} \mathfrak{h}}\left(L\left(\overline{\mathfrak{g}}_{\infty}, \bar{\Lambda}^{\mathfrak{g}}(\lambda, d)\right)\right)$ is an irreducible $\mathfrak{t} \overline{\mathfrak{g}}$-module and

$$
\mathfrak{t r}_{\mathfrak{t} \overline{\mathfrak{h}}}\left(L\left(\overline{\mathfrak{g}}_{\infty}, \bar{\Lambda}^{\mathfrak{g}}(\lambda, d)\right)\right)=L\left(\mathfrak{t} \overline{\mathfrak{g}}, \Gamma_{\mathfrak{t} \overline{\mathfrak{g}}}(\lambda, d)\right)
$$

for any partition $\lambda$ with $\lambda_{n+1}=0$ and $\overline{\mathfrak{g}}=\mathfrak{c}, \mathfrak{d}$ [CLW, Lemma 3.2]. The same result is also true for $\overline{\mathfrak{g}}=\mathfrak{a}$ and pair $\lambda=\left(\lambda^{-}, \lambda^{+}\right)$of partitions with $\lambda_{n+1}^{+}=\lambda_{m+1}^{-}=0$ by using the same arguments as in [CLW]. The anti-linear anti-involution $\omega$ on $\overline{\mathfrak{g}}_{\infty}$ induces an anti-linear anti-involution on $\mathfrak{t} \overline{\mathfrak{g}}$, which will also be denoted by $\omega$.

By a cumbersome but straightforward calculation the following theorem is a reformulation, in terms of partitions, of Theorem 2.4 and some of the results of Sections $7-9$ in [EHW].

Theorem 2.5. For $\mathfrak{g}=\mathfrak{a}, \mathfrak{c}, \mathfrak{d}$, let $\xi \in \mathfrak{h}_{\mathfrak{t} \mathfrak{g}}^{*}$.

(i) $L(\mathfrak{t} \overline{\mathfrak{a}}, \xi)$ is unitarizable with respect to $\omega$ if and only if $\xi=\Gamma_{\mathfrak{t a}}(\lambda, d)+$ $k \sum_{i=-m+1}^{n} \epsilon_{i}$ for some pair $\lambda=\left(\lambda^{+}, \lambda^{-}\right)$of partitions with $\lambda_{m}^{-}=\lambda_{n}^{+}=0$ and $d, k \in \mathbb{R}$ satisfying $d \geq \min \left\{\left(\lambda^{-}\right)_{1}^{\prime}+n-1,\left(\lambda^{+}\right)_{1}^{\prime}+m-1\right\}$, or $d \in \mathbb{Z}$ and $d \geq\left(\lambda^{-}\right)_{1}^{\prime}+\left(\lambda^{+}\right)_{1}^{\prime}$. Moreover, $N\left(\mathfrak{t} \overline{\mathfrak{a}}, \Gamma_{\mathfrak{t} \overline{\mathfrak{a}}}(\lambda, d)+k \sum_{i=-m+1}^{n} \epsilon_{i}\right)$ is irreducible for any pair $\lambda=\left(\lambda^{+}, \lambda^{-}\right)$of partitions with $\lambda_{m}^{-}=\lambda_{n}^{+}=0$ and $d, k \in \mathbb{R}$ satisfying $d>\min \left\{\left(\lambda^{-}\right)_{1}^{\prime}+n-1,\left(\lambda^{+}\right)_{1}^{\prime}+m-1\right\}$.

(ii) $L(\mathfrak{t} \overline{\mathfrak{d}}, \xi)$ is unitarizable with respect to $\omega$ if and only if $\xi=\Gamma_{\mathfrak{t} \overline{\mathfrak{d}}}(\lambda, d)$ for some partition $\lambda$ with $\lambda_{n}=0$ and $d \in \mathbb{R}$ satisfying $d \geq n-1+\lambda_{2}^{\prime}$, or $d \in \mathbb{Z}$ and $d \geq \lambda_{1}^{\prime}+\lambda_{2}^{\prime}$. Moreover, $N\left(\mathfrak{t} \overline{\mathfrak{d}}, \Gamma_{\mathfrak{t} \overline{\mathfrak{d}}}(\lambda, d)\right)$ is irreducible for every partition $\lambda$ with $\lambda_{n}=0$ and $d>n-1+\lambda_{2}^{\prime}$.

(iii) Assume that $\xi \in \mathfrak{h}_{\mathfrak{t} \mathfrak{c}}^{*}$ with $\xi\left(\widetilde{E}_{n-1}\right)=\xi\left(\widetilde{E}_{n}\right)$. Then $L(\mathfrak{t} \overline{\mathfrak{c}}, \xi)$ is unitarizable with respect to $\omega$ if and only if $\xi=\Gamma_{\mathfrak{t} \overline{\mathfrak{c}}}(\lambda, d)$ for some partition $\lambda$ with $\lambda_{n-1}=\lambda_{n}=$ 0 and $d \in \mathbb{R}$ satisfying $d \geq \frac{1}{2}\left(\lambda_{1}^{\prime}+n\right)-1$ if $n-\lambda_{1}^{\prime}$ is even; $d \geq \frac{1}{2}\left(\lambda_{1}^{\prime}+n-1\right)-1$ if $n-\lambda_{1}^{\prime}$ is odd, or $d \in \mathbb{Z}$ and $d \geq \lambda_{1}^{\prime}$. Moreover, $N\left(\mathfrak{t} \overline{\mathfrak{c}}, \Gamma_{\mathfrak{t} \overline{\mathfrak{c}}}(\lambda, d)\right)$ is irreducible for every partition $\lambda$ with $\lambda_{n-1}=\lambda_{n}=0$ and $d \in \mathbb{R}$ satisfying $d>\frac{1}{2}\left(\lambda_{1}^{\prime}+n\right)-1$ if $n-\lambda_{1}^{\prime}$ is even, and $d>\frac{1}{2}\left(\lambda_{1}^{\prime}+n-1\right)-1$ if $n-\lambda_{1}^{\prime}$ is odd.

Proposition 2.6. For $\mathfrak{g}=\mathfrak{a}, \mathfrak{c}, \mathfrak{d}$, let $L\left(\overline{\mathfrak{g}}_{\infty}, \xi\right)$ be an irreducible quasi-finite highest weight $\overline{\mathfrak{g}}_{\infty}$-module with highest weight $\xi$. Then $L\left(\overline{\mathfrak{g}}_{\infty}, \xi\right)$ is unitarizable with respect to the anti-linear anti-involution $\omega$ if and only if $\xi=\bar{\Lambda}^{\mathfrak{g}}(\lambda, d)$ for some $(\lambda, d) \in \mathcal{D}(\mathfrak{g})$.

Remark 2.7. The modules described in the proposition are modules appearing in the Howe dualities at negative levels described in [W] (cf. [LZ, Theorem 5.6, $5.8,5.9])$. 
Proof of Proposition 2.6. If $L\left(\overline{\mathfrak{g}}_{\infty}, \xi\right)$ is unitarizable, then by Remark $2.4, \xi$ satisfies $\xi\left(E_{i i}\right)=0\left(\right.$ resp. $\left.\xi\left(\widetilde{E}_{i i}\right)=0\right)$ for $|i| \gg 0$ (resp. $\left.i \gg 0\right)$ for $\overline{\mathfrak{g}}=\overline{\mathfrak{a}}($ resp. $\overline{\mathfrak{c}}, \overline{\mathfrak{d}})$. It is easy to see that $d \in \mathbb{R}$ and $\xi\left(\widetilde{E}_{i i}\right)-\xi\left(\widetilde{E}_{i+1, i+1}\right) \in \mathbb{Z}_{+}\left(\operatorname{resp.} \xi\left(E_{i i}\right)-\xi\left(E_{i+1, i+1}\right) \in \mathbb{Z}_{+}\right)$ for all $i$ (resp. $i \neq 0$ ) for $\overline{\mathfrak{g}}=\overline{\mathfrak{c}}, \overline{\mathfrak{d}}$ (resp. $\overline{\mathfrak{a}}$ ). This implies $\xi=\bar{\Lambda}^{\mathfrak{g}}(\lambda, d)$ for some partition $\lambda$ (resp. pair $\lambda=\left(\lambda^{+}, \lambda^{-}\right)$of partitions) and $d \in \mathbb{R}$ for $\overline{\mathfrak{g}}=\overline{\mathfrak{c}}, \overline{\mathfrak{d}}$ (resp. $\overline{\mathfrak{a}}$ ). Now applying the truncation functor to $L\left(\overline{\mathfrak{g}}_{\infty}, \xi\right)$ with $n \gg d$ (resp. $m, n \gg d$ ) for $\overline{\mathfrak{g}}=\overline{\mathfrak{c}}, \overline{\mathfrak{d}}$ (resp. $\overline{\mathfrak{a}}), \mathfrak{t r}_{\mathfrak{t} \mathfrak{h}}\left(L\left(\overline{\mathfrak{g}}_{\infty}, \xi\right)\right)$ is a unitarizable $\mathfrak{t} \overline{\mathfrak{g}}$-module with respect to $\omega$. By Theorem 2.5 and (2.8), we have $d \in \mathbb{Z}$ and $(\lambda, d) \in \mathcal{D}_{\mathfrak{t}}(\mathfrak{g})$. Hence $\xi=\bar{\Lambda}^{\mathfrak{g}}(\lambda, d)$ for some $(\lambda, d) \in \mathcal{D}(\mathfrak{g})$. Conversely, by Remark 2.7 the irreducible highest weight $\overline{\mathfrak{g}}_{\infty}$-modules $L\left(\overline{\mathfrak{g}}_{\infty}, \bar{\Lambda}^{\mathfrak{g}}(\lambda, d)\right)$ are unitarizable and quasi-finite.

\section{§3. Numerical data of the highest weights}

In this section, we shall provide combinatorial descriptions of $\bar{\Lambda}^{\mathfrak{g}}(\lambda, d)$ in terms of $\Lambda^{\mathfrak{g}}(\lambda, d)$.

Definition 3.1. Let $\left\{a_{i}\right\}_{i \in \mathbb{N}}$ and $\left\{b_{i}\right\}_{i \in \mathbb{N}}$ be two strictly decreasing sequences of integers (resp. half integers). They are said to form a dual pair if $\mathbb{Z}$ (resp. $1 / 2+\mathbb{Z}$ ) is the disjoint union of the sequences $\left\{a_{i}\right\}_{i \in \mathbb{N}}$ and $\left\{-b_{i}\right\}_{i \in \mathbb{N}}$.

Define the function $\rho$ on $\mathbb{N}$ by $\rho(i)=-i$ for all $i \in \mathbb{N}$. The following lemma is well known (see e.g. [M, (1.7)]).

Lemma 3.2. For any partition $\lambda$, the sequences

$$
\left\{\lambda_{i}+\rho(i)\right\}_{i \in \mathbb{N}} \quad \text { and } \quad\left\{\lambda_{i}^{\prime}+\rho(i)+\right\}_{i \in \mathbb{N}}
$$

form a dual pair.

Recall that $\phi_{\mathfrak{g}}=\sum_{i \in \mathbb{N}} \epsilon_{i}$ for $\mathfrak{g}=\mathfrak{c}, \mathfrak{d}$.

Lemma 3.3. For $\mathfrak{g}=\mathfrak{c}, \mathfrak{d}$ and $(\lambda, d) \in \mathcal{D}(\mathfrak{g})$, let $\left\{\zeta_{i}\right\}_{i \in \mathbb{N}}$ and $\left\{\bar{\zeta}_{i}\right\}_{i \in \mathbb{N}}$ be two sequences determined by

$$
\begin{aligned}
& \Lambda^{\mathfrak{g}}(\lambda, d)+\rho_{\mathfrak{g}}-d\left\langle\vartheta_{\mathfrak{g}}, K\right\rangle \phi_{\mathfrak{g}}=\sum_{i \in I_{\mathfrak{g}}} \zeta_{i} \epsilon_{i}+d \vartheta_{\mathfrak{g}}, \\
& \bar{\Lambda}^{\mathfrak{g}}(\lambda, d)+\rho_{\overline{\mathfrak{g}}}+d\left\langle\vartheta_{\mathfrak{g}}, K\right\rangle \phi_{\overline{\mathfrak{g}}}=\sum_{i \in I_{\mathfrak{g}}} \bar{\zeta}_{i} \epsilon_{i}-\frac{d\left\langle\vartheta_{\mathfrak{g}}, K\right\rangle}{\left\langle\vartheta_{\overline{\mathfrak{g}}}, K\right\rangle} \vartheta_{\overline{\mathfrak{g}}} .
\end{aligned}
$$

Then $\left\{\zeta_{i}\right\}_{i \in \mathbb{N}}$ and $\left\{\bar{\zeta}_{i}\right\}_{i \in \mathbb{N}}$ form a dual pair. Moreover, $\zeta_{i}<0$ for $i \in \mathbb{N}$ and $\mathfrak{g} \neq \mathfrak{d}$. In the case $\mathfrak{g}=\mathfrak{d}, \zeta_{i}<0$ for $i \geq 2$, and $\zeta_{1}<0$ (resp. $=0$ and $>0$ ) for $\lambda_{1}^{\prime}<d / 2$ $($ resp. $=d / 2$ and $>d / 2)$. 
Proof. By Lemma 3.2, $\left\{\zeta_{i}\right\}_{i \in \mathbb{N}}$ and $\left\{\bar{\zeta}_{i}\right\}_{i \in \mathbb{N}}$ form a dual pair. It is clear that $\zeta_{i}<0$ for $i \in \mathbb{N}$ and $\mathfrak{g}=\mathfrak{c}$. For $\mathfrak{g}=\mathfrak{d}$, we have $\lambda_{2}^{\prime} \leq d / 2$ and hence $\zeta_{i}<0$ for $i \geq 2$. Also, $\zeta_{1}=\lambda_{1}^{\prime}-d / 2<0($ resp. $=0$ and $>0)$ for $\lambda_{1}^{\prime}<d / 2($ resp. $=d / 2$ and $>d / 2)$.

Lemma 3.4. For $\mathfrak{g}=\mathfrak{c}, \mathfrak{d}$ and $(\lambda, d) \in \mathcal{D}(\mathfrak{g})$, let $\left\{\zeta_{i}\right\}_{i \in \mathbb{N}}$ and $\left\{\bar{\zeta}_{i}\right\}_{i \in \mathbb{N}}$ be the sequences defined in Lemma 3.3. Define $N(\lambda, d)=\left\{(i, j) \in \mathbb{N} \times \mathbb{N} \mid \bar{\zeta}_{i}+\bar{\zeta}_{j}=0\right.$, $i, j \in \mathbb{N}\}, J=\{j \in \mathbb{N} \mid(j, k) \notin N(\lambda, d), \forall k \in \mathbb{N}\}, \mathcal{S}=\left\{\zeta_{i} \mid i \geq 1\right\}$ and $\overline{\mathcal{S}}=\left\{\bar{\zeta}_{i} \mid i \in J\right\}$.

(i) For $\mathfrak{g}=\mathfrak{c}$, we have $\overline{\mathcal{S}}=\mathcal{S}$ and $\bar{\zeta}_{d+1}=0$.

(ii) For $\mathfrak{g}=\mathfrak{d}$, we have

$$
\begin{array}{ll}
\overline{\mathcal{S}}=\mathcal{S} \text { and } \zeta_{i} \neq 0 \neq \bar{\zeta}_{i} \text { for all } i & \text { if } d \text { is odd; } \\
\overline{\mathcal{S}} \cup\{0\}=\mathcal{S} \text { and } \zeta_{1}=0 & \text { if } d \text { is even and } \lambda_{1}^{\prime}=d / 2 ; \\
\overline{\mathcal{S}}=\mathcal{S} \text { and } \bar{\zeta}_{i}=0 \text { for some } i & \text { if } d \text { is even and } \lambda_{1}^{\prime} \neq d / 2 .
\end{array}
$$

Proof. We shall only prove the case $\mathfrak{g}=\mathfrak{d}$. The proofs of the other cases are similar and easier. For $j \geq 2$, we have $\zeta_{1}+\zeta_{j} \leq \lambda_{1}^{\prime}+\lambda_{2}^{\prime}-d-j+1 \leq-1$ and hence $\zeta_{1} \neq-\zeta_{j}$ for $j \geq 2$. Since $\left\{\zeta_{i}\right\}_{i \in \mathbb{N}}$ and $\left\{\bar{\zeta}_{i}\right\}_{i \in \mathbb{N}}$ form a dual pair, $\zeta_{1} \neq \pm \zeta_{j}$ for $j \geq 2$ and $\zeta_{i}$ are negative for $i \geq 2$, we have $\zeta_{i} \in \overline{\mathcal{S}}$ for $i \geq 2$, and $\zeta_{1} \in \overline{\mathcal{S}}$ for $\zeta_{1} \neq 0$. This implies $\overline{\mathcal{S}} \supseteq \mathcal{S} \backslash\{0\}$. For $x \in \overline{\mathcal{S}}$, we have $-x \notin \overline{\mathcal{S}}$ and hence $-x \in-\mathcal{S}$. Therefore $\overline{\mathcal{S}}=\mathcal{S} \backslash\{0\}$. By Lemma 3.2, $\mathcal{S}$ (resp. $\overline{\mathcal{S}}$ ) contains 0 if and only if $d$ is even and $\lambda_{1}^{\prime}=d / 2\left(\right.$ resp. $\left.\lambda_{1}^{\prime} \neq d / 2\right)$.

Recall that $\phi_{\mathfrak{a}}=-\sum_{i \leq 0} \epsilon_{i}$.

Lemma 3.5. For $(\lambda, d) \in \mathcal{D}(\mathfrak{a})$, let $\left\{\zeta_{i}\right\}_{i \in \mathbb{Z}}$ and $\left\{\bar{\zeta}_{i}\right\}_{i \in \mathbb{Z}}$ be two sequences determined by

$$
\begin{aligned}
\Lambda^{\mathfrak{a}}(\lambda, d)+\rho_{\mathfrak{a}}-d \phi_{\mathfrak{a}} & =\sum_{i \in \mathbb{Z}}\left(\zeta_{i}-1\right) \epsilon_{i}+d \vartheta_{\mathfrak{a}} \\
\bar{\Lambda}^{\mathfrak{a}}(\lambda, d)+\rho_{\mathfrak{a}}+d \phi_{\mathfrak{a}} & =\sum_{i \in \mathbb{Z}} \bar{\zeta}_{i} \epsilon_{i}-d \vartheta_{\mathfrak{a}}
\end{aligned}
$$

Define $N(\lambda, d)=\left\{(i, j) \in I_{\mathfrak{a}} \times I_{\mathfrak{a}} \mid \bar{\zeta}_{i}=\bar{\zeta}_{j}, i \leq 0<j\right\}, J_{+}=\{j \in \mathbb{N} \mid(i, j) \notin$ $N(\lambda, d), \forall i \leq 0\}, J_{-}=\{i \in \mathbb{Z} \mid(i, j) \notin N(\lambda, d), \forall j \in \mathbb{N}\}, \mathcal{S}_{+}=\left\{\zeta_{i} \mid i \geq 1\right\}$, $\mathcal{S}_{-}=\left\{\zeta_{i} \mid i \leq 0\right\}, \overline{\mathcal{S}}_{+}=\left\{\bar{\zeta}_{i} \mid i \in J_{+}\right\}$and $\overline{\mathcal{S}}_{-}=\left\{\bar{\zeta}_{i} \mid i \in J_{-}\right\}$. Then $\overline{\mathcal{S}}_{+}=-\mathcal{S}_{-}$ and $\overline{\mathcal{S}}_{-}=-\mathcal{S}_{+}$.

Proof. Let $\mathcal{B}_{+}=\left\{\bar{\zeta}_{i} \mid i \in \mathbb{N}\right\}$ and $\mathcal{B}_{-}=\left\{\bar{\zeta}_{i} \mid i \leq 0\right\}$. By Lemma 3.2, we have

$$
\left(-\mathcal{S}_{+}\right) \sqcup \mathcal{B}_{+}=\mathbb{Z} \quad \text { and } \quad\left(-\mathcal{S}_{-}\right) \sqcup \mathcal{B}_{-}=\mathbb{Z} .
$$


For $x \in \overline{\mathcal{S}}_{+}$, we have $x \notin \mathcal{B}_{-}$by the definition of $\overline{\mathcal{S}}_{+}$and hence $x \in-\mathcal{S}_{-}$. Therefore $\overline{\mathcal{S}}_{+} \subseteq-\mathcal{S}_{-}$. Now assume $x \in-\mathcal{S}_{-}$. We have $x \notin \mathcal{B}_{-}$. Since $\left\{\zeta_{i}\right\}_{i \in \mathbb{Z}}$ is strictly increasing, we have $x \notin-\mathcal{S}_{+}$and hence $x \in \mathcal{B}_{+}$. Thus $x \in \mathcal{B}_{+} \backslash \mathcal{B}_{-}=\overline{\mathcal{S}}_{+}$ and therefore $-\mathcal{S}_{-} \subseteq \overline{\mathcal{S}}_{+}$. Similarly, $-\mathcal{S}_{+}=\overline{\mathcal{S}}_{-}$.

We shall use the notation defined in Lemmas 3.4 and 3.5 in the rest of the paper. By (2.3) and Lemma 3.5, we have (for $(\lambda, d) \in \mathcal{D}(\mathfrak{a}), \sigma \in W_{\mathfrak{a}}$ )

$$
\begin{aligned}
\sigma^{-1}\left(\Lambda^{\mathfrak{a}}(\lambda, d)+\rho_{\mathfrak{a}}\right) & =\sum_{i \in \mathbb{Z}}\left(\zeta_{i}-1\right) \epsilon_{\sigma^{-1}(i)}+d \phi_{\mathfrak{a}}+d \vartheta_{\mathfrak{a}} \\
& =\sum_{i \in \mathbb{Z}} \zeta_{\sigma(i)} \epsilon_{i}-\sum_{i \in \mathbb{Z}} \epsilon_{i}+d \phi_{\mathfrak{a}}+d \vartheta_{\mathfrak{a}} .
\end{aligned}
$$

By Lemma 3.4 and (2.4), we have (for $(\lambda, d) \in \mathcal{D}(\mathfrak{g}), \sigma \in W_{\mathfrak{g}}$ and $\mathfrak{g}=\mathfrak{c}, \mathfrak{d}$ )

$$
\sigma^{-1}\left(\Lambda^{\mathfrak{g}}(\lambda, d)+\rho_{\mathfrak{g}}\right)=\sum_{i \in \mathbb{N}} \zeta_{\sigma(i)} \epsilon_{i}+d\left\langle\vartheta_{\mathfrak{g}}, K\right\rangle \phi_{\mathfrak{g}}+d \vartheta_{\mathfrak{g}}+d \vartheta_{\mathfrak{g}} .
$$

For $\eta$ belonging to the subspace of $\mathfrak{h}_{\overline{\mathfrak{g}}}^{*}$ spanned by the $\epsilon_{j}$ s and $\vartheta_{\overline{\mathfrak{g}}}$, let $[\eta]^{+}$

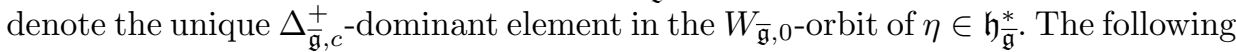
two propositions are important for proving the main theorem in the next section.

Proposition 3.6. Let $\left\{j_{i}\right\}_{i \in \mathbb{N}}$ be the strictly increasing sequence with $J=\left\{j_{i} \mid\right.$ $i \in \mathbb{N}\}$. For $(\lambda, d) \in \mathcal{D}(\mathfrak{g})$ with $\mathfrak{g}=\mathfrak{c}, \mathfrak{d}$ and a partition $\mu$ with $\Lambda^{\mathfrak{g}}(\mu, d)=\sigma^{-1} \circ$ $\Lambda^{\mathfrak{g}}(\lambda, d)$ for some $\sigma \in W_{\mathfrak{g}, k}^{0}$, we have

$$
\begin{aligned}
\bar{\Lambda}^{\mathfrak{g}}(\mu, d)+\rho_{\overline{\mathfrak{g}}}+d\left\langle\vartheta_{\mathfrak{g}}, K\right\rangle \phi_{\overline{\mathfrak{g}}} \\
= \begin{cases}{\left[\sum_{i \in \mathbb{N} \backslash J} \bar{\zeta}_{i} \epsilon_{i}+\sum_{i \in \mathbb{N}} \bar{\zeta}_{\sigma_{\sigma(i)}} \epsilon_{j_{i}}-\frac{d\left\langle\vartheta_{\mathfrak{g}}, K\right\rangle}{\left\langle\vartheta_{\overline{\mathfrak{g}}}, K\right\rangle} \vartheta_{\overline{\mathfrak{g}}}\right]^{+}} & \text {if } 0 \notin \mathcal{S}, \\
{\left[\sum_{i \in \mathbb{N} \backslash J} \bar{\zeta}_{i} \epsilon_{i}+\sum_{i \in \mathbb{N}} \bar{\zeta}_{j_{\sigma^{0}(i)}} \epsilon_{j_{i}}-\frac{d\left\langle\vartheta_{\mathfrak{g}}, K\right\rangle}{\left\langle\vartheta_{\overline{\mathfrak{g}}}, K\right\rangle} \vartheta_{\overline{\mathfrak{g}}}\right]^{+}} & \text {if } 0 \in \mathcal{S} .\end{cases}
\end{aligned}
$$

Here $\sigma^{0}$ appears only in the case $\mathfrak{g}=\mathfrak{d}$ and it is determined by $\overline{\sigma^{0}}=\sigma$ (see Lemmas 2.2 and 2.3).

Proof. In the proof, $\sqcup$ means disjoint union. Let $\left\{\xi_{i}\right\}_{i \in \mathbb{N}}$ and $\left\{\bar{\xi}_{i}\right\}_{i \in \mathbb{N}}$ be two sequences determined by

$$
\begin{aligned}
\Lambda^{\mathfrak{g}}(\mu, d)+\rho_{\mathfrak{g}}-d\left\langle\vartheta_{\mathfrak{g}}, K\right\rangle \phi_{\mathfrak{g}} & =\sum_{i \in I_{\mathfrak{g}}} \xi_{i} \epsilon_{i}+d \vartheta_{\mathfrak{g}}, \\
\bar{\Lambda}^{\mathfrak{g}}(\mu, d)+\rho_{\overline{\mathfrak{g}}}+d\left\langle\vartheta_{\mathfrak{g}}, K\right\rangle \phi_{\overline{\mathfrak{g}}} & =\sum_{i \in I_{\mathfrak{g}}} \bar{\xi}_{i} \epsilon_{i}-\frac{d\left\langle\vartheta_{\mathfrak{g}}, K\right\rangle}{\left\langle\vartheta_{\overline{\mathfrak{g}}}, K\right\rangle} \vartheta_{\overline{\mathfrak{g}}} .
\end{aligned}
$$


Assume $0 \notin \mathcal{S}$. We have $\left\{\bar{\zeta}_{j_{i}}\right\}_{i \in \mathbb{N}}=\left\{\zeta_{i}\right\}_{i \in \mathbb{N}}$ by Lemma 3.4. By Lemma 3.3, Lemma 3.4 , and the fact that $\sigma$ acts on $\mathbb{Z}^{*}$ as a signed permutation, we have

$$
\left\{-\zeta_{\sigma(i)} \mid i \in \mathbb{N}\right\} \sqcup\left\{\bar{\zeta}_{j_{\sigma(i)}} \mid i \in \mathbb{N}\right\} \sqcup\left\{\bar{\zeta}_{i} \mid i \in \mathbb{N} \backslash J\right\}=\mathbb{Z}(\text { or } 1 / 2+\mathbb{Z}) .
$$

Since $\left\{\xi_{i}\right\}_{i \in \mathbb{N}}$ and $\left\{\bar{\xi}_{i}\right\}_{i \in \mathbb{N}}$ form a dual pair, and $\left\{\xi_{i} \mid i \in \mathbb{N}\right\}=\left\{\zeta_{\sigma(i)} \mid i \in \mathbb{N}\right\}$ by (3.2), we have $\left\{\bar{\xi}_{i} \mid i \in \mathbb{N}\right\}=\left\{\bar{\zeta}_{j_{\sigma(i)}} \mid i \in \mathbb{N}\right\} \sqcup\left\{\bar{\zeta}_{i} \mid i \in \mathbb{N} \backslash J\right\}$. Therefore the proposition holds for this case since $\left\{\bar{\xi}_{i}\right\}_{i \in \mathbb{N}}$ is a decreasing sequence.

The case of $0 \in \mathcal{S}$ only occurs when $\mathfrak{g}=\mathfrak{d}$ with $\zeta_{1}=0$. We have $\left\{\bar{\zeta}_{j_{i}} \mid i \in \mathbb{N}\right\}$ $=\left\{\zeta_{i} \mid i \in \mathbb{N}\right\} \backslash\{0\}$. Since $\sigma$ acts on $\mathbb{Z}^{*}$ as a signed permutation, by Lemma 2.3 we have

$$
\left\{-\zeta_{\sigma(i)} \mid i \in \mathbb{N}\right\} \sqcup\left\{\bar{\zeta}_{j_{\sigma^{0}(i)}} \mid i \in \mathbb{N}\right\} \sqcup\left\{\bar{\zeta}_{i} \mid i \in \mathbb{N} \backslash J\right\}=\mathbb{Z}
$$

Now the proposition also follows in this case using the arguments above.

Proposition 3.7. Let $\left\{j_{i}\right\}_{i \in \mathbb{Z}}$ be the strictly decreasing sequence such that $J_{+}=$ $\left\{j_{i} \mid i \leq 0\right\}$ and $J_{-}=\left\{j_{i} \mid i \in \mathbb{N}\right\}$, and let $J=J_{-} \sqcup J_{+}$. For $(\lambda, d) \in \mathcal{D}(\mathfrak{a})$ and $a$ partition $\mu$ with $\Lambda^{\mathfrak{a}}(\mu, d)=\sigma^{-1} \circ \Lambda^{\mathfrak{a}}(\lambda, d)$ for some $\sigma \in W_{\mathfrak{a}, k}^{0}$, we have

$$
\bar{\Lambda}^{\mathfrak{a}}(\mu, d)+\rho_{\mathfrak{a}}+d \phi_{\mathfrak{a}}=\left[\sum_{i \in \mathbb{Z} \backslash J} \bar{\zeta}_{i} \epsilon_{i}+\sum_{i \in \mathbb{Z}} \bar{\zeta}_{j_{\sigma(i)}} \epsilon_{j_{i}}-d \vartheta_{\mathfrak{a}}\right]^{+} .
$$

Proof. In the proof, $\sqcup$ means disjoint union. Let $\left\{\xi_{i}\right\}_{i \in \mathbb{Z}}$ and $\left\{\bar{\xi}_{i}\right\}_{i \in \mathbb{Z}}$ be two sequences determined by

$$
\begin{aligned}
\Lambda^{\mathfrak{a}}(\mu, d)+\rho_{\mathfrak{a}}+\sum_{i \in \mathbb{Z}} \epsilon_{i}-d \phi_{\mathfrak{a}} & =\sum_{i \in \mathbb{Z}} \xi_{i} \epsilon_{i}+d \vartheta_{\mathfrak{a}} \\
\bar{\Lambda}^{\mathfrak{a}}(\mu, d)+\rho_{\mathfrak{a}}+d \phi_{\mathfrak{a}} & =\sum_{i \in \mathbb{Z}} \bar{\xi}_{i} \epsilon_{i}-d \vartheta_{\mathfrak{a}}
\end{aligned}
$$

By Lemma 3.5, we have

$$
\mathbb{Z}=\left(-\mathcal{S}_{+}\right) \sqcup\left(\overline{\mathcal{S}}_{+}\right) \sqcup\left\{\bar{\zeta}_{i} \mid i \in \mathbb{N} \backslash J_{+}\right\}=\left(-\mathcal{S}_{+}\right) \sqcup\left(-\mathcal{S}_{-}\right) \sqcup\left\{\bar{\zeta}_{i} \mid i \in \mathbb{N} \backslash J_{+}\right\} .
$$

Therefore $\mathbb{Z}=\left\{-\zeta_{\sigma(i)} \mid i \in \mathbb{Z}\right\} \sqcup\left\{\bar{\zeta}_{i} \mid i \in \mathbb{N} \backslash J_{+}\right\}$because $\sigma$ acts as a permutation on $\mathbb{Z}$. Since $\xi_{i}=\zeta_{\sigma(i)}$ for $i \in \mathbb{Z}$ by $(3.1)$ and $\zeta_{\sigma(i)}=-\bar{\zeta}_{j_{\sigma(i)}}$ for $i \in \mathbb{Z}$ by Lemma 3.5, we have

$$
\begin{aligned}
\mathbb{Z} & =\left\{-\zeta_{\sigma(i)} \mid i \in \mathbb{N}\right\} \sqcup\left\{-\zeta_{\sigma(i)} \mid i \leq 0\right\} \sqcup\left\{\bar{\zeta}_{i} \mid i \in \mathbb{N} \backslash J_{+}\right\} \\
& =\left\{-\xi_{i} \mid i \in \mathbb{N}\right\} \sqcup\left\{\bar{\zeta}_{j_{\sigma(i)}} \mid i \leq 0\right\} \sqcup\left\{\bar{\zeta}_{i} \mid i \in \mathbb{N} \backslash J_{+}\right\} .
\end{aligned}
$$

Since $\left\{\xi_{i}\right\}_{i \in \mathbb{N}}$ and $\left\{\bar{\xi}_{i}\right\}_{i \in \mathbb{N}}$ form a dual pair, we have $\left\{\bar{\xi}_{i} \mid i \in \mathbb{N}\right\}=\left\{\bar{\zeta}_{j_{\sigma(i)}} \mid i \leq 0\right\}$ $\sqcup\left\{\bar{\zeta}_{i} \mid i \in \mathbb{N} \backslash J_{+}\right\}$. Similarly, $\left\{\bar{\xi}_{i} \mid i \leq 0\right\}=\left\{\bar{\zeta}_{j_{\sigma(i)}} \mid i \in \mathbb{N}\right\} \sqcup\left\{\bar{\zeta}_{i} \mid i \in\right.$ 
$\left.\left(-\mathbb{Z}_{+}\right) \backslash J_{-}\right\}$. Therefore the proposition holds since $\left\{\bar{\xi}_{i}\right\}_{i \in \mathbb{N}}$ is a decreasing sequence and $\left\{\bar{\xi}_{-i}\right\}_{i \in \mathbb{Z}_{+}}$is an increasing sequence.

\section{§4. $\mathfrak{u}_{\mathfrak{g}}^{-}$-homology formulas for $\overline{\mathfrak{g}}_{\infty}$-modules}

In this section we give a combinatorial proof of Enright's $\mathfrak{u}_{\overline{\mathfrak{g}}}^{-}$-homology formula [E] for the unitarizable highest weight $\overline{\mathfrak{g}}_{\infty}$-modules with highest weight $\bar{\Lambda}^{\mathfrak{g}}(\lambda, d)$.

For a module $V$ over a Lie algebra $\mathcal{G}$, let $\mathrm{H}_{k}(\mathcal{G} ; V)$ denote $k$-th homology group of $\mathcal{G}$ with coefficients in $V$. It is well known that the homology groups $\mathrm{H}_{k}\left(\mathfrak{u}_{\overline{\mathfrak{g}}}^{-} ; V\right)$ are $\mathfrak{l}_{\overline{\mathfrak{g}}}$-modules. The $\mathfrak{u}_{\overline{\mathfrak{g}}}^{-}$-homology of unitarizable highest weight modules is described by the following theorem which was obtained in [CK, Theorem 7.2] for $\overline{\mathfrak{g}}_{\infty}=\mathfrak{a}_{\infty}$ and in [CKW, Theorem 6.5] for $\overline{\mathfrak{g}}_{\infty}=\mathfrak{c}_{\infty}, \mathfrak{d}_{\infty}$. The theorem holds for a more general situation by using the correspondence of homology groups in the sense of super duality [CLW, Theorem 4.10] together with Kostant's formulas for integrable $\mathfrak{g}_{\infty}$-modules (cf. [J, Ko, V, CK]).

Theorem 4.1. For $(\lambda, d) \in \mathcal{D}(\mathfrak{g})$ with $\mathfrak{g}=\mathfrak{c}, \mathfrak{d}$ (resp. $\mathfrak{g}=\mathfrak{a})$, we have, as $\mathfrak{l}_{\overline{\mathfrak{g}}}$ modules,

$$
\mathrm{H}_{k}\left(\mathfrak{u}_{\overline{\mathfrak{g}}}^{-} ; L\left(\overline{\mathfrak{g}}_{\infty}, \bar{\Lambda}^{\mathfrak{g}}(\lambda, d)\right)\right) \cong \bigoplus_{\mu} L\left(\mathfrak{l}_{\overline{\mathfrak{g}}}, \bar{\Lambda}^{\mathfrak{g}}(\mu, d)\right),
$$

where the sum is over all partitions (resp. pairs of partitions) $\mu$ such that $\Lambda^{\mathfrak{g}}(\mu, d)$ $=w^{-1} \circ \Lambda^{\mathfrak{g}}(\lambda, d)$ for some $w \in W_{\mathfrak{g}, k}^{0}$.

For $\xi$ belonging to the subspace of $\mathfrak{h}_{\mathfrak{g}}^{*}$ spanned by the $\epsilon_{j}$ s and $\vartheta_{\overline{\mathfrak{g}}}$, let $\Psi(\xi)=$ $\left\{\alpha \in \Delta_{\overline{\mathfrak{g}}, n}^{+} \mid\left(\xi+\rho_{\overline{\mathfrak{g}}} \mid \alpha\right)=0\right\}$ and define $\Phi(\xi)$ to be the subset of $\Delta_{\overline{\mathfrak{g}}, n}^{+}$consisting of all roots $\beta$ satisfying the following conditions [E, DES]:

(i) $\left\langle\xi+\rho_{\overline{\mathfrak{g}}}, \beta^{\vee}\right\rangle \in \mathbb{N}$;

(ii) $(\beta \mid \alpha)=0$ for all $\alpha \in \Psi(\xi)$;

(iii) $\beta$ is short if $\Psi(\xi)$ contains a long root.

Let $W_{\overline{\mathfrak{g}}}(\xi)$ be the subgroup of $W_{\overline{\mathfrak{g}}}$ that is generated by the reflections $s_{\beta}$ with $\beta \in \Phi(\xi)$. Define $\Delta_{\overline{\mathfrak{g}}}(\xi)$ to be the subset of $\Delta_{\overline{\mathfrak{g}}}$ consisting of the roots $\vartheta \in \Delta_{\overline{\mathfrak{g}}}$ such that $s_{\vartheta}$ lies in $W_{\overline{\mathfrak{g}}}(\xi)$.

For $(\lambda, d) \in \mathcal{D}(\mathfrak{g})$, let $\Delta_{\overline{\mathfrak{g}}}(\lambda, d)=\Delta_{\overline{\mathfrak{g}}}\left(\bar{\Lambda}^{\mathfrak{g}}(\lambda, d)\right)$ and $W_{\overline{\mathfrak{g}}}(\lambda, d)=W_{\overline{\mathfrak{g}}}\left(\bar{\Lambda}^{\mathfrak{g}}(\lambda, d)\right)$. Then $\Delta_{\overline{\mathfrak{g}}}(\lambda, d)$ is an abstract root system and $W_{\overline{\mathfrak{g}}}(\lambda, d)$ is the Weyl group of $\Delta_{\overline{\mathfrak{g}}}(\lambda, d)\left[\mathrm{E}, \mathrm{EHW}\right.$ ( see also Lemma 4.2 below). Let $\Delta_{\overline{\mathfrak{g}}}^{+}(\lambda, d)=\Delta_{\overline{\mathfrak{g}}}(\lambda, d) \cap \Delta_{\overline{\mathfrak{g}}}^{+}$ be the set of positive roots of $\Delta_{\overline{\mathfrak{g}}}(\lambda, d)$. Set $W_{\overline{\mathfrak{g}}, 0}(\lambda, d)=W_{\overline{\mathfrak{g}}}(\lambda, d) \cap W_{\overline{\mathfrak{g}}, 0}$. Let $W_{\overline{\mathfrak{g}}}^{0}(\lambda, d)$ denote the set of minimal length representatives of the left coset space $W_{\overline{\mathfrak{g}}}(\lambda, d) / W_{\overline{\mathfrak{g}}, 0}(\lambda, d)$ and let $W_{\overline{\mathfrak{g}}, k}^{0}(\lambda, d)$ be the subset of $W_{\overline{\mathfrak{g}}}^{0}(\lambda, d)$ consisting of all elements $\sigma$ with $\ell_{(\lambda, d)}(\sigma)=k$, where $\ell_{(\lambda, d)}$ is the length function on $W_{\overline{\mathfrak{g}}}(\lambda, d)$. 
For $(\lambda, d) \in \mathcal{D}(\mathfrak{g})$, let $J^{0}=J \sqcup\left\{j \mid \bar{\zeta}_{j}=0\right\}$ for $\mathfrak{g}=\mathfrak{c}, \mathfrak{d}$ and define

$\Upsilon(\lambda, d)$

$$
= \begin{cases}\left\{\epsilon_{i}-\epsilon_{j} \in \Delta_{\overline{\mathfrak{g}}}^{+} \mid i \in J_{-}, j \in J_{+}\right\} & \text {for } \mathfrak{g}=\mathfrak{a} ; \\ \left\{-\epsilon_{i}-\epsilon_{j} \in \Delta_{\overline{\mathfrak{g}}}^{+} \mid i<j, i, j \in J^{0}\right\} & \text { for } \mathfrak{g}=\mathfrak{c} ; \\ \left\{-\epsilon_{i}-\epsilon_{j} \in \Delta_{\mathfrak{g}}^{+} \mid i<j, i, j \in J\right\} & \text { if } J^{0} \neq J \text { or } d / 2 \notin \mathbb{Z}, \text { for } \mathfrak{g}=\mathfrak{d} ; \\ \left\{-\epsilon_{i}-\epsilon_{j},-2 \epsilon_{i} \in \Delta_{\overline{\mathfrak{g}}}^{+} \mid i<j, i, j \in J\right\} & \text { if } J^{0}=J \text { and } d / 2 \in \mathbb{Z}, \text { for } \mathfrak{g}=\mathfrak{d} .\end{cases}
$$

Lemma 4.2. For $(\lambda, d) \in \mathcal{D}(\mathfrak{g})$, we have

$$
\begin{aligned}
& \Delta_{\overline{\mathfrak{g}}}(\lambda, d) \\
& = \begin{cases}\left\{\epsilon_{i}-\epsilon_{j} \in \Delta_{\overline{\mathfrak{g}}} \mid i \neq j, i, j \in J_{-} \sqcup J_{+}\right\} & \text {for } \mathfrak{g}=\mathfrak{a} ; \\
\left\{ \pm\left( \pm \epsilon_{i}-\epsilon_{j}\right) \in \Delta_{\overline{\mathfrak{g}}} \mid i<j, i, j \in J^{0}\right\} & \text { for } \mathfrak{g}=\mathfrak{c} ; \\
\left\{ \pm\left( \pm \epsilon_{i}-\epsilon_{j}\right) \in \Delta_{\overline{\mathfrak{g}}} \mid i<j, i, j \in J\right\} & \text { if } J^{0} \neq J \text { or } d / 2 \notin \mathbb{Z}, \text { for } \mathfrak{g}=\mathfrak{d} ; \\
\left\{ \pm\left( \pm \epsilon_{i}-\epsilon_{j}\right), \pm 2 \epsilon_{i} \in \Delta_{\overline{\mathfrak{g}}} \mid i<j, i, j \in J\right\} & \text { if } J^{0}=J \text { and } d / 2 \in \mathbb{Z}, \text { for } \mathfrak{g}=\mathfrak{d} .\end{cases}
\end{aligned}
$$

Proof. For $(\lambda, d) \in \mathcal{D}(\mathfrak{g})$, we have $\Phi\left(\bar{\Lambda}^{\mathfrak{g}}(\lambda, d)\right) \subseteq \Upsilon(\lambda, d)$ by Lemmas 3.4 and 3.5. Using the relations of the Weyl groups, it is easy to observe that $\Upsilon(\lambda, d) \subseteq \Delta_{\bar{g}}(\lambda, d)$. Now the lemma follows by using the relations of the Weyl groups again.

Lemma 4.3. For $(\lambda, d) \in \mathcal{D}(\mathfrak{g})$, there is a bijection from $W_{\mathfrak{g}, k}^{0}$ to $W_{\overline{\mathfrak{g}}, k}^{0}(\lambda, d)$.

Proof. By Lemma 4.2, it is clear that $W_{\mathfrak{g}, k}^{0}=W_{\overline{\mathfrak{g}}, k}^{0}(\lambda, d)$ for the cases $\mathfrak{g}=\mathfrak{a}$ and $\mathfrak{g}=\mathfrak{d}$ with $J^{0} \neq J$ or $d / 2 \notin \mathbb{Z}$. For $\mathfrak{g}=\mathfrak{c}$ and $\mathfrak{g}=\mathfrak{d}$ with $J^{0}=J$ and $d / 2 \in \mathbb{Z}$, the conclusion follows from Lemmas 4.2 and 2.2 .

Using Theorem 4.1, Proposition 3.6, Proposition 3.7, Lemma 4.3 and Lemma 2.3 together with (2.3) and (2.4), we obtain the following theorem.

Theorem 4.4. For $(\lambda, d) \in \mathcal{D}(\mathfrak{g})$ and $k \in \mathbb{Z}_{+}$, we have, as $\mathfrak{l}_{\overline{\mathfrak{g}}}$-modules,

$$
\mathrm{H}_{k}\left(\mathfrak{u}_{\overline{\mathfrak{g}}}^{-} ; L\left(\overline{\mathfrak{g}}_{\infty}, \bar{\Lambda}^{\mathfrak{g}}(\lambda, d)\right)\right) \cong \bigoplus_{w \in W_{\overline{\mathfrak{g}}, k}^{0}(\lambda, d)} L\left(\mathfrak{l}_{\overline{\mathfrak{g}}},\left[w^{-1}\left(\bar{\Lambda}^{\mathfrak{g}}(\lambda, d)+\rho_{\overline{\mathfrak{g}}}\right)\right]^{+}-\rho_{\overline{\mathfrak{g}}}\right) .
$$

Remark 4.5. There is a counterpart of Theorem 4.11 of [CLW] for $\mathfrak{u}^{+}$-cohomology in the sense of [Liu, Section 4]. The analogous statement is also true for $\mathfrak{g}=\mathfrak{a}$. The formulas for $\mathfrak{u}^{+}$-cohomology can be proved by the same argument as in [CLW]. Therefore, there is an analogue of Theorem 4.4 for $\mathfrak{u}_{\mathfrak{g}}^{+}$-cohomology in the sense of [Liu]. The formulas for cohomology can be proved by the same argument as above. 


\section{$\S 5$. Homology formulas for unitarizable modules over finite-dimensional Lie algebras}

In this section we shall give a new proof of Enright's homology formulas for unitarizable modules over classical Lie algebras corresponding to the three Hermitian symmetric pairs of classical types, $(S U(p, q), S U(p) \times S U(q)),(S p(n, \mathbb{R}), U(n))$ and $\left(S O^{*}(2 n), U(n)\right)$.

For $\xi$ belonging to $\mathfrak{h}_{\mathfrak{t} \mathfrak{g}}^{*}$, let $\Psi(\xi)=\left\{\alpha \in \Delta_{\mathfrak{t} \overline{\mathfrak{g}}, n}^{+} \mid\left(\xi+\rho_{\mathfrak{t} \overline{\mathfrak{g}}} \mid \alpha\right)=0\right\}$ and define $\Phi(\xi)$ to be the subset of $\Delta_{\mathfrak{t} \overline{\mathfrak{g}}, n}^{+}$consisting of the roots $\beta$ satisfying the following conditions $[\mathrm{E}, \mathrm{DES}]$ :

(i) $\left\langle\xi+\rho_{\mathfrak{t} \overline{\mathfrak{g}}}, \beta^{\vee}\right\rangle \in \mathbb{N}$;

(ii) $(\beta \mid \alpha)=0$ for all $\alpha \in \Psi(\xi)$;

(iii) $\beta$ is short if $\Psi(\xi)$ contains a long root.

Let $W_{\mathfrak{t} \overline{\mathfrak{g}}}(\xi)$ be the subgroup of $W_{\mathfrak{t} \overline{\mathfrak{g}}}$ that is generated by the reflections $s_{\beta}$ with $\beta \in \Phi(\xi)$. Associated to $W_{\mathfrak{t} \overline{\mathfrak{g}}}(\xi)$, let $\Delta_{\mathfrak{t} \overline{\mathfrak{g}}}(\xi)$ denote the subset of $\Delta_{\mathfrak{t} \overline{\mathfrak{g}}}$ consisting of the roots $\vartheta$ such that $s_{\vartheta}$ lies in $W_{\mathfrak{t} \overline{\mathfrak{g}}}(\xi)$. We also let $[\xi]^{+}$be the unique $\Delta_{\mathfrak{t} \overline{\mathfrak{g}}, c^{-}}^{+}$ dominant element in the $W_{\mathfrak{t} \overline{\mathfrak{g}}, 0}$-orbit of $\xi$.

Assume that the irreducible module $L(\mathfrak{t} \mathfrak{\mathfrak { g }}, \xi)$ is unitarizable with highest weight $\xi \in \mathfrak{h}_{\mathfrak{t} \mathfrak{g}}^{*}$. Then $\Delta_{\mathfrak{t} \overline{\mathfrak{g}}}(\xi)$ is an abstract root system and $W_{\mathfrak{t} \overline{\mathfrak{g}}}(\xi)$ is the Weyl group of $\Delta_{\mathfrak{t} \overline{\mathfrak{g}}}(\xi)$ by $\left[\mathrm{E}, \mathrm{EHW}\right.$. Let $\Delta_{\mathfrak{t} \overline{\mathfrak{g}}}^{+}(\xi)=\Delta_{\mathfrak{t} \overline{\mathfrak{g}}}(\xi) \cap \Delta_{\mathfrak{t} \overline{\mathfrak{g}}}^{+}$be the set of positive roots of $\Delta_{\mathfrak{t} \overline{\mathfrak{g}}}(\xi)$. Set $W_{\mathfrak{t} \overline{\mathfrak{g}}, 0}(\xi)=W_{\mathfrak{t} \overline{\mathfrak{g}}}(\xi) \cap W_{\mathfrak{t} \overline{\mathfrak{g}}, 0}$. Let $W_{\mathfrak{t} \mathfrak{g}}^{0}(\xi)$ denote the set of minimal length representatives of the left coset space $W_{\mathfrak{t} \overline{\mathfrak{g}}}(\xi) / W_{\mathfrak{t} \overline{\mathfrak{g}}, 0}(\xi)$ and let $W_{\mathbf{t} \mathfrak{g}, k}^{0}(\xi)$ be the subset of $W_{\mathfrak{t} \overline{\mathfrak{g}}}^{0}(\xi)$ consisting of all elements $\sigma$ with $\ell_{\xi}(\sigma)=k$, where $\ell_{\xi}$ is the length function on $W_{\mathfrak{t} \overline{\mathfrak{g}}}(\xi)$.

Theorem 5.1. For $\overline{\mathfrak{g}}=\mathfrak{a}, \mathfrak{c}$ or $\mathfrak{d}$, let $L(\mathfrak{t} \overline{\mathfrak{g}}, \xi)$ be a unitarizable $\mathfrak{t} \overline{\mathfrak{g}}-$ module with highest weight $\xi \in \mathfrak{h}_{\mathfrak{t g}}^{*}$. Assume that $\xi$ satisfies the assumption of case (iii) of Theorem 2.5 (cf. case (ii) of [EHW, Theorem 9.4]) for $\mathfrak{t} \overline{\mathfrak{g}} \cong \mathfrak{s o}(2 n)$. For $k \in \mathbb{Z}_{+}$, we have, as $\mathfrak{l}_{\mathfrak{t} \overline{\mathfrak{g}}}$-modules,

$$
\mathrm{H}_{k}\left(\mathfrak{u}_{\mathfrak{t} \overline{\mathfrak{g}}}^{-} ; L(\mathfrak{\mathfrak { t } \mathfrak { g }}, \xi)\right) \cong \bigoplus_{w \in W_{\mathfrak{t} \overline{\mathfrak{g}}, k}^{0}(\xi)} L\left(\mathfrak{l}_{\mathfrak{t} \overline{\mathfrak{g}}},\left[w^{-1}\left(\xi+\rho_{\mathfrak{t} \overline{\mathfrak{g}}}\right)\right]^{+}-\rho_{\mathfrak{t} \overline{\mathfrak{g}}}\right) .
$$

Proof. Since

$$
\mathrm{H}_{i}\left(\mathfrak{u}_{\mathfrak{t} \overline{\mathfrak{a}}}^{-} ; L\left(\mathfrak{t} \overline{\mathfrak{a}}, \mu+k \sum_{i=-m+1}^{n} \epsilon_{i}\right)\right)=\mathrm{H}_{i}\left(\mathfrak{u}_{\mathfrak{t} \overline{\mathfrak{a}}}^{-} ; L(\mathfrak{t} \overline{\mathfrak{a}}, \mu)\right) \otimes L\left(\mathfrak{l}_{\mathfrak{t} \overline{\mathfrak{a}}}, k \sum_{i=-m+1}^{n} \epsilon_{i}\right)
$$

for all $i \geq 0, k \in \mathbb{C}$ and $\mu \in \mathfrak{h}_{\mathfrak{t a}}^{*}$, it is sufficient to consider all $\xi$ with $k=0$ appearing in case (i) of Theorem 2.5 when $\overline{\mathfrak{g}}=\overline{\mathfrak{a}}$. 
First we assume that $\xi=\Gamma_{\mathfrak{t} \overline{\mathfrak{g}}}(\lambda, d)$ with $d \notin \mathbb{Z}$. Then we have $\Delta_{\mathfrak{t} \overline{\mathfrak{g}}}(\xi)=\emptyset$ and $L(\mathfrak{t} \overline{\mathfrak{g}}, \xi)=N(\mathfrak{t} \overline{\mathfrak{g}}, \xi)$ by Theorem 2.5. Therefore $L(\mathfrak{t} \overline{\mathfrak{g}}, \xi)$ is a free $\mathfrak{u}_{\mathfrak{t} \overline{\mathfrak{g}}}^{-}$-module and hence $H_{k}\left(\mathfrak{u}_{\mathfrak{t} \overline{\mathfrak{g}}}^{-} ; L(\mathfrak{t} \overline{\mathfrak{g}}, \xi)\right)=L\left(\mathfrak{l}_{\mathfrak{t} \overline{\mathfrak{g}}}, \xi\right)($ resp. $=0)$ for $k=0$ (resp. $\left.k>0\right)$. Thus the theorem holds for this case.

Now we assume that $\xi=\Gamma_{\mathfrak{t} \overline{\mathfrak{g}}}(\lambda, d)$ for some $(\lambda, d) \in \mathcal{D}_{\mathfrak{t}}(\mathfrak{g})$. By a direct calculation, we have $\Delta_{\mathfrak{t} \overline{\mathfrak{g}}}(\xi)=\Delta_{\overline{\mathfrak{g}}}(\lambda, d) \cap \Delta_{\mathfrak{t} \overline{\mathfrak{g}}}$. Recall that $\mathfrak{t r}_{\mathfrak{t} \mathfrak{\mathfrak { h }}}\left(L\left(\overline{\mathfrak{g}}_{\infty}, \bar{\Lambda}^{\mathfrak{g}}(\lambda, d)\right)\right)=$ $L\left(\mathfrak{t} \overline{\mathfrak{g}}, \Gamma_{\mathfrak{t} \overline{\mathfrak{g}}}(\lambda, d)\right)$ for $(\lambda, d) \in \mathcal{D}_{\mathfrak{t}}(\mathfrak{g})$. Since $\mathfrak{t r}_{\mathfrak{t} \overline{\mathfrak{h}}}\left(L\left(\overline{\mathfrak{g}}_{\infty}, \bar{\Lambda}^{\mathfrak{g}}(\lambda, d)\right)\right)=L\left(\mathfrak{t} \overline{\mathfrak{g}}, \Gamma_{\mathfrak{t} \overline{\mathfrak{g}}}(\lambda, d)\right)$ and homology commutes with the truncation functor, we have

$$
\mathrm{H}_{k}\left(\mathfrak{u}_{\mathfrak{t} \mathfrak{g}}^{-} ; L(\mathfrak{t} \overline{\mathfrak{g}}, \xi)\right)=\mathfrak{t r}_{\mathfrak{t h}}\left(\mathrm{H}_{k}\left(\mathfrak{u}_{\overline{\mathfrak{g}}}^{-} ; L\left(\overline{\mathfrak{g}}_{\infty}, \bar{\Lambda}^{\mathfrak{g}}(\lambda, d)\right)\right)\right) .
$$

Note that $H_{k}\left(\mathfrak{u}_{\overline{\mathfrak{g}}}^{-} ; L\left(\overline{\mathfrak{g}}_{\infty}, \bar{\Lambda}^{\mathfrak{g}}(\lambda, d)\right)\right)$ with $k \geq 0$ decomposes into the direct sum of irreducible $\mathfrak{l}_{\mathfrak{g}}$-modules of the form $L\left(\mathfrak{l}_{\overline{\mathfrak{g}}}, \bar{\Lambda}^{\mathfrak{g}}(\mu, d)\right)$ for some partition $\mu$ (resp. pair $\mu=\left(\mu^{-}, \mu^{+}\right)$of partitions) if $\mathfrak{g}=\mathfrak{c}, \mathfrak{d}($ resp. $\mathfrak{a})$ and $\mathfrak{t r}_{\mathfrak{t h}}\left(L\left(\mathfrak{l}_{\mathfrak{g}}, \bar{\Lambda}^{\mathfrak{g}}(\mu, d)\right)\right)=$ $L\left(\mathfrak{l}_{\mathfrak{t} \overline{\mathfrak{g}}}, \Gamma_{\mathfrak{t} \overline{\mathfrak{g}}}(\mu, d)\right)$. Therefore, the theorem also holds in this case by Theorem 4.4.

Remark 5.2. By Remark 4.5, Enright's cohomology formulas for unitarizable modules over classical Lie algebras with highest weights satisfying the assumption in the theorem above can be proved in the same manner as above.

\section{Acknowledgements}

The second author is very grateful to Shun-Jen Cheng for numerous discussions and useful suggestions. He also thanks Weiqiang Wang for valuable suggestions. The first and second authors were partially supported by an NSC-grant and thank NCTS/South. The third author thanks NCTS/South for hospitality and support.

\section{References}

[BB] A. Bjorner and F. Brenti, Combinatorics of Coxeter groups, Grad. Texts in Math. 231, Springer, New York, 2005. Zbl 1110.05001 MR 2133266

[BrS] J. Brundan and C. Stroppel, Highest weight categories arising from Khovanov's diagram algebras IV: the general linear supergroup, J. Eur. Math. Soc. 14 (2012), 373-419.

[CK] S.-J. Cheng and J.-H. Kwon, Howe duality and Kostant homology formula for infinitedimensional Lie superalgebras, Int. Math. Res. Notices 2008, art. ID rnn 085, 52 pp. Zbl 1217.17011 MR 2439556

[CKW] S.-J. Cheng, J.-H. Kwon and W. Wang, Kostant homology formulas for oscillator modules of Lie superalgebras, Adv. Math. 224 (2010), 1548-1588. Zbl 1210.17026 MR 2646304

[CL] S.-J. Cheng and N. Lam, Irreducible characters of the general linear superalgebra and super duality, Comm. Math. Phys. 298 (2010), 645-672. Zbl 1217.17004 MR 2670923

[CLW] S.-J. Cheng, N. Lam and W. Wang, Super duality and irreducible characters of orthosymplectic Lie superalgebras, Invent. Math. 183 (2011), 189-224. Zbl pre05852689 MR 2755062 
[CW] S.-J. Cheng and W. Wang, Brundan-Kazhdan-Lusztig and super duality conjectures, Publ. RIMS Kyoto Univ. 44 (2008), 1219-1272. Zbl 1210.17009 MR 2477902

[CWZ] S.-J. Cheng, W. Wang and R. B. Zhang, Super duality and Kazhdan-Lusztig polynomials, Trans. Amer. Math. Soc. 360 (2008) 5883-5924. Zbl pre05358271 MR 2425696

[DES] M. Davidson, T. J. Enright and R. Stanke, Differential operators and highest weight representations. Mem. Amer. Math. Soc. 94 (1991), no. 455, iv+102 pp. Zbl 0759.22015 MR 1081660

[E] T. J. Enright, Analogues of Kostant's u-cohomology formula for unitary highest weight modules, J. Reine Angew. Math. 392 (1988), 27-36. Zbl 0651.17003 MR 0965055

[EHW] T. J. Enright, R. Howe and N. R. Wallach, A classification of unitary highest weight modules, in Representation theory of reductive groups, Birkhäuser, Boston, 1983, 97143. Zbl 0535.22012 MR 0733809

[H1] R. Howe, Remarks on classical invariant theory, Trans. Amer. Math. Soc. 313 (1989), 539-570. Zbl 0674.15021 MR 0986027

[H2] , Perspectives on invariant theory: Schur duality, multiplicity-free actions and beyond, in The Schur lectures (Tel Aviv, 1992), Israel Math. Conf. Proc. 8, Bar-Ilan Univ., 1995, 1-182. Zbl 0844.20027 MR 1321638

[J] E. Jurisich, An exposition of generalized Kac-Moody algebras, in Lie algebras and their representations (Seoul, 1995), Contemp. Math. 194, Amer. Math. Soc., Providence, RI, 1996, 121-159. Zbl 0867.17016 MR 1395597

[K] V. Kac, Infinite-dimensional Lie algebras, 3rd ed., Cambridge Univ. Press, Cambridge, 1990. Zbl 0716.17022

[KR] V. Kac and A. Radul, Quasi-finite highest weight modules over the Lie algebra of differential operators on the circle, Comm. Math. Phys. 157 (1993), 429-457. Zbl 0826.17027 MR 1104219

[Ko] B. Kostant, Lie algebra cohomology and the generalized Borel-Weil theorem, Ann. of Math. 74 (1961), 329-387. Zbl 0134.03501 MR 0142696

$[\mathrm{Ku}] \quad$ S. Kumar, Kac-Moody groups, their flag varieties and representation theory, Progr. Math. 204, Birkhäuser Boston, Boston, MA, 2002. Zbl 1026.17030 MR 1923198

[LZ] N. Lam and R. B. Zhang, Quasi-finite modules for Lie superalgebras of infinite rank, Trans. Amer. Math. Soc. 358 (2006), 403-439. Zbl 1105.17013 MR 2171240

[Liu] L. Liu, Kostant's formula for Kac-Moody Lie algebras, J. Algebra 149 (1992), 155-178. Zbl 0779.17024 MR 1165205

[M] I. G. Macdonald, Symmetric functions and Hall polynomials, Oxford Math. Monogr., Clarendon Press, Oxford, 1995. Zbl 0824.05059 MR 1354144

[V] D. A. Vogan, Representation of real reductive Lie groups, Progr. Math. 15, Birkhäuser, 1981. Zbl 0469.22012 MR 0632407

[W] W. Wang, Duality in infinite dimensional Fock representations, Comm. Contemp. Math. 1 (1999), 155-199. Zbl 0951.17011 MR 1696098 